\title{
Subharmonic capillary-gravity waves in large containers subject to horizontal vibrations
}

\author{
José M. Perez-Gracia, Jeff Porter, Fernando Varas and José M. Vega†
}

This paper deals with nearly inviscid, capillary-gravity, modulated waves parametrically excited by monochromatic horizontal vibrations in liquid containers whose width and depth are both large compared with the wavelength of the excited waves. A general linear amplitude equation is derived with appropriate boundary conditions that provides the threshold acceleration and associated spatiotemporal patterns, which compare very well with experimental measurements and visualizations. The primary instability is associated with a pair of complex Floquet multipliers that are close to (but strictly different from) -1 , meaning that the instability is not strictly (2:1) subharmonic. The resulting (quasi-periodic) waves are generally oblique, not perpendicular to the vibrating endwalls. The extension of the theory to other confined systems such as vibrating containers of arbitrary shape and vibrating drops is also considered.

Key words: capillary waves, parametric instability, pattern formation

\section{Introduction}

The effect of external vibrations on fluid systems is of interest in many scientific and engineering applications such as liquid storage, mixing, pattern formation and the study of fluid instabilities. The case of vertical vibrations, first considered by Faraday (1831), has become a paradigm of parametric excitation (Fauve 1995). This is because, in addition to experimental accessibility, it admits a fairly clean theoretical analysis under the assumption of a perfectly flat horizontal free surface undergoing perfectly vertical oscillations (Benjanin \& Ursell 1954).

Horizontally vibrating systems are more resistant to analysis. Direct excitation produces harmonic waves with the forcing frequency at arbitrary small forcing amplitude. Parametric excitation is triggered by the harmonic field and requires that the forcing acceleration exceeds a threshold value; the resulting waves can be periodic, with a frequency equal to, e.g., one-half of the forcing frequency (as in the Faraday system), or quasi-periodic, exhibiting two frequencies whose sum coincides with the forcing frequency. The latter will also be called subhamonic below, even though the wave frequencies need not be an exact fraction of the forcing frequency. Experimental and theoretical analyses have concentrated on two distinguished limits. 
(a)

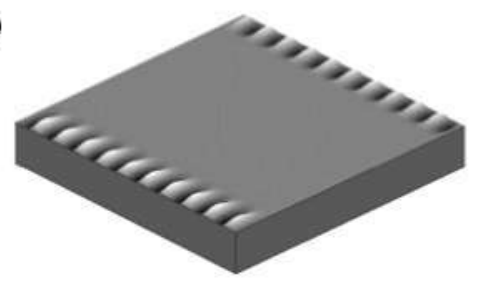

(c)

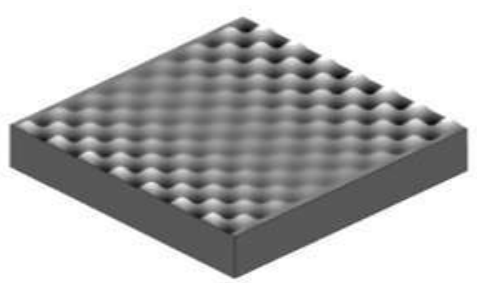

(b)

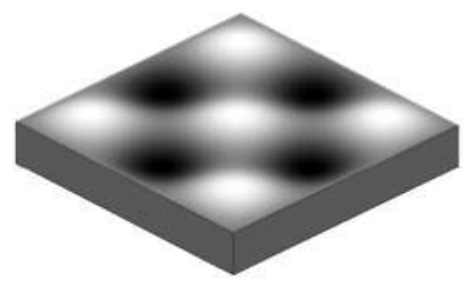

(d)

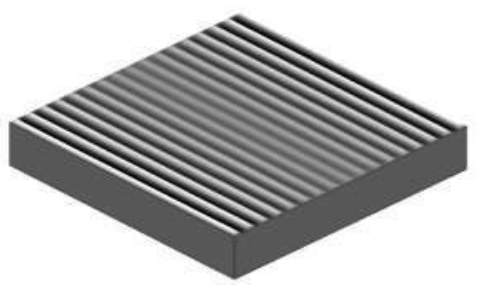

FIGURE 1. (a) Cross-waves, $(b)$ sloshing modes, $(c)$ oblique subharmonic waves and $(d)$ harmonic wavetrains.

Cross-waves (see figure 1) are strictly (2:1) subharmonic waves produced by a wavemaker consisting in a partially submerged horizontally vibrating plate (Barnard \& Pritchard 1972; Miles \& Henderson 1990). They were already described by Faraday (1831) and theoretically analysed by Garrett (1970). Subsequent studies, initiated by Jones (1984), focused on gravitational waves, assuming wave crests perpendicular to the wavemaker. Cross-waves in a semi-infinite rectangular tank can be analysed using the Havelock (1929) solution for the wavemaker problem, which leads to approximations based on the nonlinear Schrödinger equation with the forcing mechanism in the boundary conditions (see Bernoff, Kwok \& Lichter (1989), Shemer \& Lichter (1990) and references therein). Oblique waves were also considered in this context by Shemer \& Chamesse (1999), who retained capillary effects and attributed obliqueness to side band instabilities.

Interaction between both endwalls produces sloshing modes (see figure 1) that exhibit new dynamics promoted by wave reflection at the endwalls. Both harmonic and subharmonic sloshing modes have been considered, among others, by Miles (1984), Funakoshi \& Inoue (1988), Feng (1997) and Hill (2003). Faltinsen, Timokha, and collaborators (in a series of papers, see Faltinsen, Rognebakke \& Timokha 2006; Hermann \& Timokha 2008 and references therein) have considered fully nonlinear gravity waves under general excitation (not necessarily horizontal) using modal expansions; Frandsen (1997) and Wu \& Chen (2009), instead, addressed this problem using finite differences. For parametric excitation resulting from horizontal vibration of large containers in the gravity limit, the wave crests can be expected to be perpendicular to the endwalls, which is consistent with the above-mentioned property of cross-waves. On the other hand, obliquely oriented subharmonic waves (see figure 1), whose crests are not perpendicular to the endwalls, have been experimentally observed by Porter et al. (2012) in horizontally vibrated containers using driving frequencies for which capillary effects cannot be neglected. Similar capillary-gravity oblique waves have been observed in related configurations (Taneda 1994; Moisy et al. 2012). These suggest that obliqueness could be unavoidable when capillary effects are present, a conjecture that which will be checked in the analysis below. In fact, the main purpose of this paper is to construct a consistent linear 
theory able to predict both the subharmonic instability threshold and the resulting patterns in horizontally vibrated containers. The general capillary-gravity limit will be considered and no particular orientations will be assumed; purely gravitational waves and cross-waves will appear as particular limits. The main difficulty associated with capillary effects is due to uncertainties in modelling contact line motion, which could contaminate quantitative comparison with experiments. Empirical models depending on tunable parameters are not satisfactory in a theory derived from first principles. Here, instead, the contact line will be pinned to the edge of the sidewalls and endwalls, which can be experimentally achieved by either filling up the container in a brimful way or using hydrophobic coatings.

Parametric excitation is triggered by the harmonic wave field produced by the vibrating endwalls, which exhibits two distinguished components. On the one hand, two counterpropagating harmonic wavetrains (see figure 1) aligned along the vibrating endwalls are present that propagate (and decay by viscous dissipation) inwards from the endwalls. On the other hand, an oscillatory bulk flow (OBF) is also present that is essentially different from the wavetrains because:

(i) it is slowly varying in space, while the wavetrains show an $O(1)$ wavelength;

(ii) it is purely inviscid and penetrates from the endwalls a distance comparable to the container depth, while penetration of the wavetrains is determined by viscous dissipation;

(iii) it exhibits a spatially constant pressure at the free surface, thus being independent of both gravity and capillary forces, while the wavetrains do depend on these.

Property (ii) means that the OBF is the dominant forcing mechanism if the container depth is at least somewhat large compared with the viscous decay length of the subharmonic wavetrains. This requires that viscosity not be too small, consistent with the experimentally accessible conditions with ordinary liquids when the container width and depth are comparable and of the order of tens of centimetres and the forcing frequency is larger than $10 \mathrm{~Hz}$. This will be the limit considered in this paper.

The OBF was called the local oscillation by Havelock (1929) in his analysis of harmonic gravity waves produced by wavemakers and it was therefore present in subsequent works on cross-waves that used the Havelock solution; for example, Jones (1984) called it the local disturbance. This flow has been formulated in terms of a linear equation with well-defined boundary conditions by two of us (Varas \& Vega 2007 ) in the case of two-dimensional, horizontally vibrated containers. The OBF produces a pressure field that is the counterpart of the spatially uniform, temporally periodic vertical-pressure-gradient field produced by inertial forces in the Faraday experiment. The essential difference is that the vertical pressure gradient produced by the OBF is not spatially uniform.

Selection of the subharmonic wave pattens at threshold is determined by the $\mathrm{OBF}$ and the boundary conditions. The usual approach in rectangular containers is to decompose the patterns into plane waves. Here, instead, a new approach will be undertaken in which no particular pattern is assumed. Specifically, a purely twodimensional, linear evolution equation for the free surface elevation will be derived that has substantial interest in itself. It will be referred to below as a general amplitude equation since it is independent of the container geometry and allows for any possible spatially modulated pattern composed by waves in arbitrary orientations. The boundary conditions account for wave reflection at the sidewalls and endwalls and, to the best of the authors' knowledge, are not available in the literature for fixed contact line. Thus, they will be derived in appendix B, using the Fourier transform 


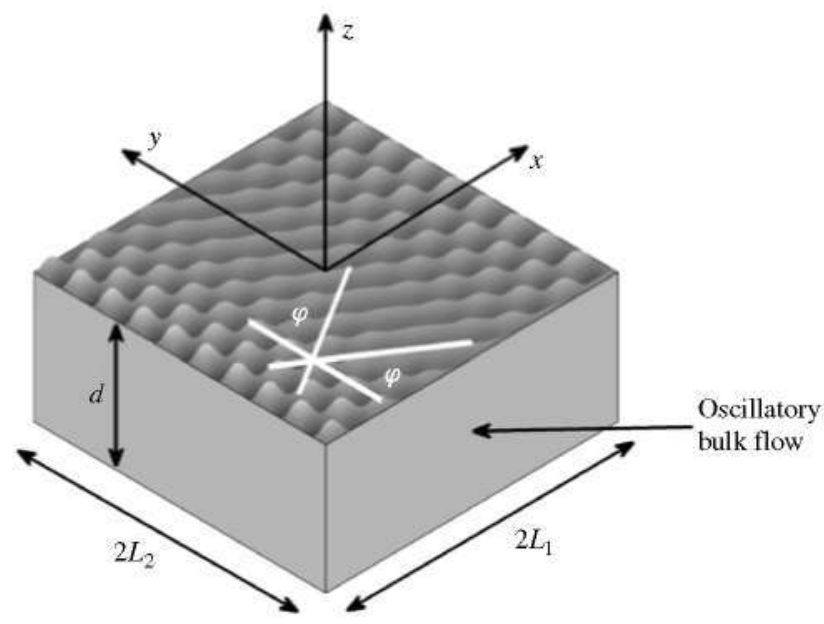

FIGURE 2. Sketch of the vibrating container.

of the local solution in a region near the container walls. This general amplitude equation and boundary conditions provide both the subharmonic threshold amplitude and the critical eigenfunctions. It turns out that the primary subharmonic instability is associated with a pair of complex conjugate Floquet multipliers that are close to (but not equal to) -1 , with eigenfunctions exhibiting a generally oblique eigenvector. Thus, the threshold patterns are oblique, slowly modulated (quasi-periodic) waves that are nearly standing in the short time scale and show activity that slowly drifts back and forth between the two endwalls (type I patterns in figure $6, \S 4.2$ ). Interaction with nearby modes (which are nearly marginal because the container is large) leads to secondary bifurcations very soon after threshold. The resulting patterns are either analogous to those at threshold or exhibit similar activity near both endwalls (type II patterns in figure $6, \S 4.2$ ). The quasi-periodicity of the primary patterns and the subsequent mode interaction giving qualitatively different patterns are remarkable differences with Faraday waves. The calculated threshold amplitude compares very well with the experimental measurements by Porter et al. (2012) and also with some new experiments presented here to further check the theory. The resulting patterns also agree well with the experiments qualitatively.

With these ideas in mind, the remainder of the paper is organized as follows. The problem is formulated and the OBF is calculated in $\$ 2$, where the governing equations are simplified using a quasi-potential approximation. Section 3 is devoted to the derivation of the general amplitude equation, which is used in $\$ 4$ to obtain effective threshold conditions for the subharmonic instability. Specific results in $\$ 5$ include comparison with experiments and insights into the dependence of the threshold amplitude and patterns on the various non-dimensional parameters. A general formulation (for more general confined vibrating fluid systems) of both the OBF and the general amplitude equation is addressed in $\S 6$, where some concluding remarks are also provided.

\section{Mathematical formulation and preliminary analysis}

We consider (figure 2) a rectangular container of depth $d^{*}$ and horizontal crosssection $2 L_{1}^{*} \times 2 L_{2}^{*}$, which is vibrated horizontally in the $2 L_{1}^{*}$ direction with amplitude $a^{*}$ 
and frequency $2 \omega^{*}$; the superscript * hereafter denotes dimensional quantities. In order to account for both the gravity and capillary limits, non-dimensionalization is done with the characteristic time $\omega^{*-1}$ and length $k^{*-1}$, specifically

$$
t=\omega^{*} t^{*}, \quad d=k^{*} d^{*}, \quad L_{1}=k^{*} L_{1}^{*}, \quad L_{2}=k^{*} L_{2}^{*}, \quad a=k^{*} a^{*},
$$

where the wavenumber $k^{*}$ is defined by the capillary-gravity dispersion relation

$$
\omega^{* 2}=g k^{*}+\sigma k^{* 3} / \rho \text {. }
$$

Here, $g$ is the gravitational acceleration, $\sigma$ is the surface tension and $\rho$ is the density, all assumed constant. Viscous and capillary-gravity effects are then accounted for in terms of the modified Ohnesorge number and the gravity-capillary balance parameter,

$$
C_{g}=\mu k^{* 2} /\left(\rho \omega^{*}\right) \text { and } S=\sigma k^{* 2} /\left(\sigma k^{* 2}+\rho g\right) \text {. }
$$

respectively, where $\mu$ is the viscosity. Note that $0 \leqslant S \leqslant 1$, with the extreme values $S=0$ and 1 corresponding to the pure gravity and capillary limits, respectively.

In a static Cartesian coordinate system with the $x y$ plane containing the unperturbed free surface, the $x$ - and $y$-axes along the sidewalls and endwalls, respectively, and the $z$-axis pointing upwards, the non-dimensional continuity and momentum equations are

$$
\begin{gathered}
\nabla \cdot u+\partial_{z} w=0 . \\
\partial_{t} u-w\left(\nabla w-\partial_{z} u\right)+\left(\nabla \cdot u^{\perp}\right) u^{\perp}=-\nabla p+C_{g}\left(\Delta u+\partial_{z z} u\right), \\
\partial_{t} w+u \cdot\left(\nabla w-\partial_{\bar{c}} u\right)=-\partial_{z} p+C_{g}\left(\Delta w+\partial_{z z} w\right) .
\end{gathered}
$$

Here, $\boldsymbol{u}$ and $w$ are the horizontal and vertical velocity components, respectively, $p=$ pressure $+(1-S) z+\left(|u|^{2}+w^{2}\right) / 2$ is the hydrostatic stagnation pressure, $\partial_{t}$, $\partial_{\varepsilon}, \partial_{z}$, etc., denote partial derivatives, and

$$
\Delta=\partial_{x x}+\partial_{y y} \text { and } \nabla=e_{x} \partial_{x}+e_{y} \partial_{y}
$$

are the horizontal Laplacian and gradient operators, with $e_{x}$ and $e_{y}$ unit vectors in the $x$ and $y$ directions. The superscript ${ }^{\perp}$ indicates $90^{\circ}$ counterclockwise rotation, namely

$$
u^{\perp}=-v e_{x}+u e_{y} \quad \text { if } u=u e_{x}+v e_{y} .
$$

The convective terms in (2.5)-(2.6) are the horizontal and vertical components of the non-potential part of the three-dimensional convective term, $-\boldsymbol{v} \times \tilde{\nabla} \times \boldsymbol{v}(\tilde{\nabla}$ and $v$ denote the three-dimensional gradient operator and velocity vector, respectively).

Equations (2.4)-(2.6) apply in the domain occupied by the liquid, which is

$$
-L_{1}+a \cos 2 t<x<L_{1}+a \cos 2 t, \quad-L_{2}<y<L_{2}, \quad-d<z<f .
$$

where $a>0$ is the non-dimensional vibrating amplitude and $f$ is the free surface elevation. The boundary conditions

$$
\begin{gathered}
u=-2 a e_{x} \sin 2 t, \quad w=0 \quad \text { at } x= \pm L_{1}+a \cos 2 t, \text { at } y= \pm L_{2} \text { and at } z=-d, \\
f=0 \text { at } x= \pm L_{1}+a \cos 2 t \text { and at } y= \pm L_{2}, \\
w=\partial_{t} f+u \cdot \nabla f, \\
p-\left(|u|^{2}+w^{2}\right) / 2-(1-S) f+S \nabla \cdot\left[\nabla f /\left(1+|\nabla f|^{2}\right)^{1 / 2}\right] \\
=C_{g}\left\{2 \partial_{z} w-2\left(\partial_{z} u+\nabla w\right) \cdot \nabla f+\nabla f \cdot\left(\nabla u+\nabla u^{\top}\right) \cdot \nabla f\right\} /\left(1+|\nabla f|^{2}\right), \\
\partial_{z} u+\nabla w=\left(\nabla u+\nabla u^{\top}\right) \cdot \nabla f-\left[2 \partial_{z} w-\left(\partial_{z} u+\nabla w\right) \cdot \nabla f\right] \nabla f \quad \text { at } z=f,
\end{gathered}
$$

enforce no slip at the solid boundaries, kinematic compatibility and the balance of normal and tangential stresses at the free surface. The superscript $T$ denotes the 
transpose. Note that in the boundary condition (2.11), as anticipated, the free surface is pinned to the upper edge of the container walls. The right-hand side of (2.13) is the viscous normal stress, $C_{g}\left[\left(\tilde{\nabla} \boldsymbol{v}+\tilde{\nabla} \boldsymbol{v}^{\top}\right) \cdot \boldsymbol{n}\right] \cdot \boldsymbol{n}$, where $\boldsymbol{n}$ is the upwards unit normal to the free surface. Similarly, the boundary condition (2.14) imposes zero tangential stress, namely $\left[\left(\tilde{\nabla} \boldsymbol{v}+\tilde{\nabla} \boldsymbol{v}^{\top}\right) \cdot \boldsymbol{n}\right] \times \boldsymbol{n}=\mathbf{0}$. The problem (2.4)-(2.6), (2.10)-(2.14) is invariant under reflection about the symmetry planes of the container, namely under the actions

$$
x \rightarrow-x, \boldsymbol{u} \cdot \boldsymbol{e}_{x} \rightarrow-\boldsymbol{u} \cdot \boldsymbol{e}_{x}, t \rightarrow t+\pi / 2 \text { and } y \rightarrow-y, \boldsymbol{u} \cdot \boldsymbol{e}_{y} \rightarrow-\boldsymbol{u} \cdot \boldsymbol{e}_{y},
$$

referred to as the spatiotemporal reflection symmetry and the purely spatial reflection symmetry, respectively.

The main assumptions in this paper are

$$
L_{1}, L_{2}, d \gg 1, \quad a, C_{g} \ll 1, \quad C_{g} d \gg 1 \quad \text { and } \quad\left(\sqrt{C_{g}}+S\right)\left(L_{1}+L_{2}\right) \gg 1 .
$$

The first assumption affirms that the size of the container is much larger than the capillary-gravity wavelength (which is $2 \pi$ after the non-dimensionalization above). This is equivalent to the assumption that the forcing frequency is large compared with the frequency of the first capillary-gravity sloshing mode of the container. The assumption $a \ll 1$ is consistent with assuming that the steepness of the waves is small, i.e.

$$
|\boldsymbol{u}| \ll 1, \quad|w| \ll 1, \quad|p| \ll 1, \quad|f| \ll 1 .
$$

The last two assumptions in (2.16) (further discussed in $\S 3$ and appendix B) justify neglecting nonlinear interaction with the counterpropagating, harmonic wavetrains and viscous dissipation in the Stokes layers attached to the sidewalls and endwalls.

\subsection{Quasi-potential approximation}

Because $C_{g} \ll 1$, the oscillatory motion is nearly inviscid and a quasi-potential approximation can be applied. Here, we follow the formulation of Zhang \& Viñals (1997); see also Joseph, Funada \& Wang (2007) for related approximations. It should be noted that viscous mean flows produced by the oscillatory boundary layers require the usual assumption that the flow is potential in the bulk to be corrected (Vega, Rüdiger \& Viñals 2004), but mean flows can be ignored in the present linear approximation. Thus, the motion is inviscid in the bulk (outside the oscillatory boundary layers), where the vorticity and the Laplacian of the velocity are both zero. Thus, $\partial_{z} \boldsymbol{u}-\nabla w=\partial_{z z} \boldsymbol{u}+\Delta \boldsymbol{u}=\mathbf{0}, \boldsymbol{\nabla} \cdot \boldsymbol{u}^{\perp}=\partial_{z z} w+\Delta w=0$, and (2.4)-(2.6) simplify to

$$
\boldsymbol{\nabla} \cdot \boldsymbol{u}+\partial_{z} w=0, \quad \partial_{t} \boldsymbol{u}=-\nabla p, \quad \partial_{t} w=-\partial_{z} p \quad \text { if } z<0 .
$$

Eliminating the velocity in these equations yields

$$
\Delta p+\partial_{z z} p=0 \text { if } z<0,
$$

and invoking (2.18), the boundary conditions (2.10)-(2.11) are linearized around $x= \pm L_{1}$ and rewritten as

$$
\begin{gathered}
\partial_{x} p+a \partial_{x x} p \cos 2 t=4 a \cos 2 t, \quad f+a \partial_{x} f \cos 2 t=0 \quad \text { at } x= \pm L_{1}, \\
\partial_{y} p=0, \quad f=0 \quad \text { at } y= \pm L_{2}, \quad \partial_{z} p=0 \quad \text { at } z=-d .
\end{gathered}
$$

On the other hand, using (2.16)-(2.17) and retaining only terms up to quadratic order in the small quantities $\boldsymbol{u}, w, p, f, a$ and $C_{g}$, the boundary conditions (2.12)-(2.13) 
lead to

$$
\begin{gathered}
\partial_{f} f-w=-\nabla \cdot(f \boldsymbol{u}), \\
p-(1-S) f+S \Delta f=2 C_{g} \partial_{z} w+\left(|\boldsymbol{u}|^{2}+w^{2}\right) / 2-f \partial_{z} p \quad \text { at } z=0 .
\end{gathered}
$$

For consistency, two additional equations must be added for the velocity components at the unperturbed free surface, $\boldsymbol{u}$ and $w$. These two equations are

$$
\partial_{t} \boldsymbol{u}=-\nabla p, \quad \partial_{t} w=-\partial_{z} p+2 C_{g} \Delta w \quad \text { at } z=0,
$$

which coincide with the corresponding equations considered by Zhang \& Viñals (1997), except that the quasi-potential approximation is written here in terms of the pressure instead of the velocity potential. The derivation of the evolution equation (2.24) (in particular, ensuring that no additional quadratic terms result from convective terms) is subtle and, for completeness, is provided in appendix A.

The quasi-potential approximation (2.19)-(2.24) is the relevant one in the remainder of the paper. Even though the analysis below concentrates in the linear approximation, quadratic terms have been retained in (2.23) because they are needed to obtain the parametric forcing terms (resulting from products of terms associated with the OBF and the subharmonic waves) in the general amplitude equation.

\subsection{The oscillatory bulk flow}

The OBF is a linear harmonic flow produced by the vibrating endwalls. As anticipated in the Introduction, a crucial property of this flow is that the flow variables (denoted hereafter with the superscript $o b f$ ) vary slowly in space. In particular,

$$
\left|\nabla p^{o b f}\right| \sim\left|\partial_{z} p^{o b f}\right| \ll\left|p^{o b f}\right| .
$$

At leading order (as $a \rightarrow 0$ ), the associated pressure field is written as

$$
p^{o b f}=a P^{o b f}(x, y, z) \cos 2 t,
$$

while the remaining flow variables are such that

$$
\left|f^{o b f}\right| \sim\left|w^{o b f}\right| \sim\left|\boldsymbol{u}^{o b f}\right| \ll\left|p^{o b f}\right|,
$$

as is readily obtained, invoking (2.25), from the first boundary condition (2.23) and (2.24). Using these and substituting (2.26) into (2.19)-(2.21) and into the second boundary condition (2.23), then retaining only the leading $O(a)$ terms, yield

$$
\begin{gathered}
\Delta P^{o b f}+\partial_{z z} P^{o b f}=0 \quad \text { in }|x|<L_{1},|y|<L_{2},-d<z<0, \\
\partial_{x} P^{o b f}=4 \quad \text { at } x= \pm L_{1}, \quad \partial_{y} P^{o b f}=0 \quad \text { at } y= \pm L_{2}, \\
\partial_{z} P^{o b f}=0 \quad \text { at } z=-d, \quad P^{o b f}=0 \quad \text { at } z=0 .
\end{gathered}
$$

Note the zero-pressure boundary condition at the unperturbed free surface, $z=0$, which results from (2.27) and is a specific property of the OBF.

Owing to the Neumann boundary conditions at the sidewalls, $y= \pm L_{2}$, the unique solution to (2.28)-(2.30) is independent of $y$ and is readily obtained in closed form,

$$
P^{o b f}=\frac{32 d}{\pi^{2}} \sum_{m>0, o d d} \frac{\sinh [m \pi x /(2 d)] \cos [m \pi(z+d) /(2 d)]}{m^{2} \cosh \left[m \pi L_{1} /(2 d)\right] \sin (m \pi / 2)},
$$

where it has been taken into account that

$$
\sum_{m>0, \text { odd }} \frac{4}{m \pi} \frac{\cos [m \pi(z+d) /(2 d)]}{\sin (m \pi / 2)}=1 \text { if }-d<z<0 .
$$



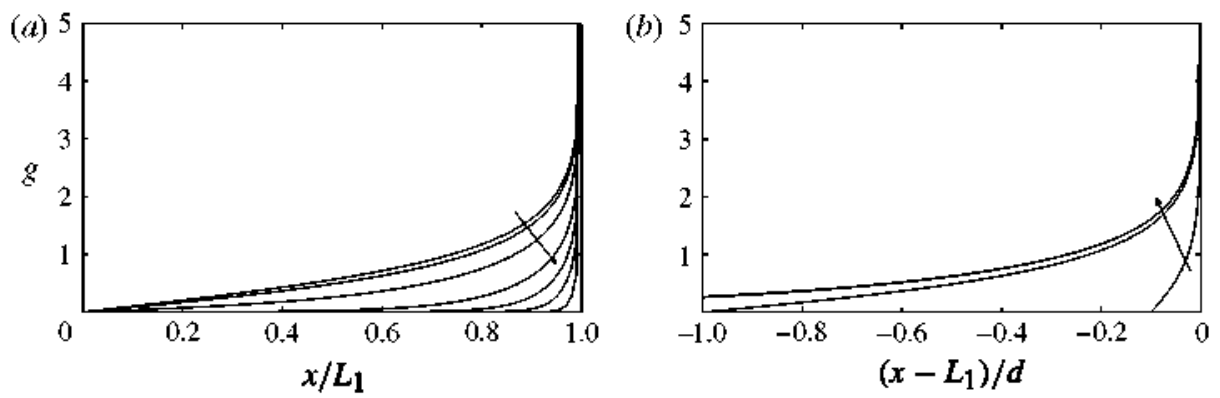

FIGURE 3. The forcing function $g$ versus $(a) x / L_{1}$ and $(b)\left(x-L_{1}\right) / d$ for $L_{1} / d=0.1,1,2,5,10,20$ and 50 ; arrows indicate increasing values of $L_{1} / d$.

The associated velocity components at $z=0$ and the free surface elevation are readily calculated from (2.22) and (2.24) (not used above), as

$$
\left(\partial_{z} p^{o b f}, f^{o b f}, w^{o b f}\right)=a g(x)(-4 \cos 2 t,-\cos 2 t, 2 \sin 2 t), \quad u^{o b f}=\mathbf{0} \quad \text { at } z=0 .
$$

where the forcing function $g$ is given by

$$
g(x)=-\partial_{2} P^{o b f} / 4 \equiv \frac{4}{\pi} \sum_{m>0, o d d} \frac{\sinh [m \pi x /(2 d)]}{m \cosh \left[m \pi L_{1} /(2 d)\right]} .
$$

Note that $g(x)$ (and thus also $\partial_{i} p^{o b f}, w^{o b f}$ and $f^{o b f}$ ) diverge logarithmically as $x \rightarrow \pm L_{1}$, but this weak singularity is consistent with the behaviour of the harmonic flow in an $O$ (1) region near the upper edge of the endwalls (namely, $\left|x \pm L_{1}\right| \sim|z| \sim 1$ ), as checked by Varas \& Vega (2007). The function $g$ is antisymmetric,

$$
g(-x)=-g(x)
$$

due to the spatiotemporal reflection symmetry in (2.15). Also, if $d \ll L_{1}$, then $g(x)$ vanishes exponentially except in two $O(d)$ regions near the endwalls. In particular, near $x=L_{1}$ (the approximation near $x=-L_{1}$ is obtained using (2.35)), $g$ behaves as

$$
g(x)=G(\zeta) \equiv \frac{4}{\pi} \sum_{m>0, \text { odd }} \frac{1}{m} \exp (-m \pi \zeta / 2) \quad \text { with } \zeta=\left(x-L_{1}\right) / d .
$$

The function $g$ is illustrated in figure 3 for several representative values of $L_{1} / d$. Note that when $g$ is plotted versus $\left(x-L_{1}\right) / d$ (right plot), only three curves are distinguished; the remaining curves coalesce to the approximation $(2,36)$ as $L_{1} / d \geqslant 2$. This is because the error of the approximation scales as $\mathrm{e}^{-\pi L_{1} / d}$.

\section{General amplitude equation for subharmonic waves}

In this section, we derive a linear amplitude equation for the free surface elevation that provides the slow temporal evolution of subharmonic waves. This equation is obtained from the simplified equations $(2.19)-(2.24)$, after expanding the flow variables as

$$
\begin{gathered}
(p, f)=\left(p^{o b f}, f^{o b f}\right)+\left[\mathrm{e}^{\mathrm{i} f}\left(P^{s}, F^{s}\right)+c . c .\right]+\cdots \\
(\boldsymbol{u}, \boldsymbol{w})=\left(\boldsymbol{u}^{o b f}, \boldsymbol{w}^{o b f}\right)+\left[\mathrm{e}^{\mathrm{i} f}\left(\boldsymbol{U}^{s}, W^{s}\right)+c . c .\right]+\cdots
\end{gathered}
$$


where c.c. stands for the complex conjugate. The harmonic OBF is as calculated in the last section and the subharmonic flow, denoted with the superscript $s$, is such that

$$
\begin{aligned}
\left|\partial_{t} P^{s}\right| & \ll\left|P^{s}\right| \ll 1, \quad\left|\partial_{t} F^{s}\right| \ll\left|F^{s}\right| \ll 1, \\
\left|\partial_{t} \boldsymbol{U}^{s}\right| & \ll\left|\boldsymbol{U}^{s}\right| \ll 1, \quad\left|\partial_{t} W^{s}\right| \ll\left|W^{s}\right| \ll 1 .
\end{aligned}
$$

These conditions imply a separation of temporal scales. Substituting (3.1)-(3.2) into (2.24) and setting the coefficient of $\mathrm{e}^{\mathrm{it}}$ to zero lead to

$$
\partial_{t} \boldsymbol{U}^{s}+\mathrm{i} \boldsymbol{U}^{s}=-\nabla P^{s}, \quad \partial_{t} W^{s}+\mathrm{i} W^{s}=-\partial_{z} P^{s}+2 C_{g} \Delta W^{s},
$$

which, to the approximation relevant here (recall that $\partial_{t}$ is small), leads to

$$
\boldsymbol{U}^{s} \simeq \mathrm{i} \nabla P^{s}-\partial_{t} \nabla P^{s}, \quad W^{s} \simeq \mathrm{i} \partial_{z} P^{s}-\partial_{t z} P^{s}+2 C_{g} \partial_{z} \Delta P^{s} .
$$

Substituting these and the expressions in (2.33) for the OBF into (3.2), then the resulting equation and (3.1) into (2.19)-(2.23), setting to zero the coefficient of $\mathrm{e}^{\mathrm{it}}$ in the resulting equations and retaining only up to quadratic order in the small quantities $P^{s}, F^{s}, C_{g}$ and $a$, lead to the following formulation

$$
\left.\begin{array}{c}
\Delta P^{s}+\partial_{z z} P^{s}=0 \quad \text { in }-\infty<z<0, \\
\partial_{z} P^{s}-F^{s}=-\mathrm{i} \partial_{t} F^{s}-\mathrm{i} \partial_{z t} P^{s}+2 \mathrm{i} C_{g} \partial_{z} \Delta P^{s}+a g(x) \Delta \bar{P}^{s} / 2, \\
P^{s}-(1-S) F^{s}+S \Delta F^{s}=2 \mathrm{i} C_{g} \partial_{z z} P^{s}+3 a g(x) \partial_{z} \bar{P}^{s} / 2 \quad \text { at } z=0,
\end{array}\right\}
$$

Here, the function $g(x)$ is as given by (2.34) and the overbar indicates complex conjugation. The boundary condition (3.8) is obtained by noting that the subharmonic surface waves only affect a horizontal layer of $O(1)$ thickness attached to the free surface (the subharmonic flow is at rest below this layer).

Equations (3.6)-(3.8) provide the linear dynamics of subharmonic surface waves, including the leading-order effects of viscous damping and parametric forcing, accounted for in those terms proportional to $C_{g} \ll 1$ and $a \ll 1$, respectively. This three-dimensional formulation can be reduced to a two-dimensional description by taking advantage of the fact that this problem is nearly singular. At leading order, setting $a=C_{g}=0$ and neglecting time derivatives, equations (3.6)-(3.8) reduce to

$$
\begin{gathered}
\Delta P^{s}+\partial_{z z} P^{s}=0 \quad \text { in }-\infty<z<0, \\
\partial_{z} P^{s}-F^{s}=P^{s}-(1-S) F^{s}+S \Delta F^{s}=0 \quad \text { at } z=0, \\
\partial_{z} P^{s} \rightarrow 0 \text { as } z \rightarrow-\infty .
\end{gathered}
$$

This unforced problem is singular since it admits non-zero solutions of the form

$$
\left(P^{s}, F^{s}\right)=\sum_{j} A_{j}(x, y, t)\left(\mathrm{e}^{z}, 1\right) \mathrm{e}^{\mathrm{i}\left(v_{j} x+\kappa_{j} y\right)} \quad \text { with } \sqrt{v_{j}^{2}+\kappa_{j}^{2}}=1,
$$

which, invoking (3.1), represent combinations of plane travelling wavetrains. In fact, when the whole container (whose horizontal dimension is large) is considered, the complex amplitudes $A_{j}$ and the unit wavevectors $\boldsymbol{k}_{j}=v_{j} \boldsymbol{e}_{x}+\kappa_{j} \boldsymbol{e}_{y}$ must be allowed to vary slowly both in space (horizontal directions) and in time. This is the usual (quite involved) approach to treating nearly inviscid surface waves in three space dimensions in the literature. It generally requires considering a number of amplitude equations for the evolution of the amplitudes of the wavetrains. These equations are typically 
coupled, even in the linear approximation, because of reflection at the endwalls and sidewalls. Wave reflection also implies that this approach depends in a critical way on geometry, meaning that the whole analysis must be customized for particular geometries. For instance, circular waves (instead of plane waves) should be considered in circular containers.

As an alternative, a broader yet more subtle approach is followed here that provides a general amplitude equation, which is two-dimensional and independent of the geometry. As a first step in deriving this equation, we note that if the complex amplitudes $A_{j}$ and the wavenumbers $\left|\boldsymbol{k}_{j}\right| \equiv 1$ are both constant, the wave fields (3.12) are such that

$$
\partial_{t} F^{s}=0, \quad \Delta P^{s}+P^{s}=0, \quad \Delta F^{s}+F^{s}=0 .
$$

Viscous damping and parametric forcing promote slow spatial variations of both $A_{j}$ and the $\boldsymbol{k}_{j}$, which means that the exact equalities (3.13) must be replaced by

$$
\left|\partial_{t} F^{s}\right| \ll 1, \quad\left|\Delta P^{s}+P^{s}\right| \ll\left|P^{s}\right|, \quad\left|\Delta F^{s}+F^{s}\right| \ll\left|F^{s}\right| .
$$

These resonance conditions define slowly modulated patterns and implicitly imply a separation of scales in both space and time. Non-resonant patterns, not satisfying (3.14), decay to zero on the fast time scale $t \sim 1$ and can be ignored.

As a second step, equation (3.12) is written as

$$
\left(P^{s}, F^{s}\right)=\left(\mathrm{e}^{z}, 1\right) F^{s}(x, y, t),
$$

where $F^{s}=\sum A_{j} \mathrm{e}^{\mathrm{i}\left(v_{j} x+\kappa_{j} y\right)}$ satisfies (3.14). The general amplitude equation for $F^{s}$ is obtained by rewriting (3.15) as

$$
\left(P^{s}, F^{s}\right)=\left(\mathrm{e}^{z}, 1\right) F^{s}(x, y, t)+\left(P_{1}^{s}, F_{1}^{s}\right)+\mathrm{NRT},
$$

where the new terms account for viscous damping, parametric forcing, and spatiotemporal modulations, which were neglected in (3.9)-(3.11). NRT denotes nonresonant terms, not satisfying (3.14). Instead, $P_{1}^{s}$ and $F_{1}^{s}$ are resonant and must satisfy

$$
\left|\partial_{t} P_{1}^{s}\right|,\left|\Delta P_{1}^{s}+P_{1}^{s}\right| \ll\left|P_{1}^{s}\right| \ll\left|P^{s}\right|, \quad\left|\partial_{t} F_{1}^{s}\right|,\left|\Delta F_{1}^{s}+F_{1}^{s}\right| \ll\left|F^{s}\right|,
$$

Substituting (3.16) into (3.6)-(3.8) and invoking (3.14) and (3.17) yield

$$
\left.\begin{array}{c}
\partial_{z z} P_{1}^{s}-P_{1}^{s}=-\left(\Delta F^{s}+F^{s}\right) \mathrm{e}^{z} \quad \text { in }-\infty<z<0, \\
\partial_{z} P_{1}^{s}-F_{1}^{s}=-2 \mathrm{i} \partial_{t} F^{s}+2 \mathrm{i} C_{g} \Delta F^{s}+a g(x) \Delta \bar{F}^{s} / 2, \\
P_{1}^{s}-F_{1}^{s}=-S\left(\Delta F^{s}+F^{s}\right)+2 \mathrm{i} C_{g} F^{s}+3 a g(x) \bar{F}^{s} / 2 \quad \text { at } z=0 \\
\partial_{z} P_{1}^{s}=0 \quad \text { at } z=-\infty .
\end{array}\right\}
$$

This problem is singular (and thus not generally solvable) because its homogeneous part (setting to zero $a, C_{g}$ and $F^{s}$ ) coincides exactly with (3.9)-(3.11), which admits the non-trivial solutions (3.12). Thus, a solvability condition must be imposed and this provides the evolution equation for $F^{s}$. The solvability condition results from multiplying (3.18) by $\mathrm{e}^{z}$, integrating in $-\infty<z<0$, integrating by parts, and imposing the boundary condition $(3.20)$, which yield

$$
\partial_{z} P_{1}^{s}-P_{1}^{s}=-\left(\Delta F^{s}+F^{s}\right) / 2 \text { at } z=0 .
$$

Substituting into this the boundary conditions (3.19) leads to

$$
\partial_{t} F^{s}+\mathrm{i}(1+2 S)\left(\Delta F^{s}+F^{s}\right) / 4=C_{g}\left(\Delta F^{s}-F^{s}\right)+\mathrm{i} a g(x)\left(3 \bar{F}^{s}-\Delta \bar{F}^{s}\right) / 4 .
$$


Note that since $C_{g}, a$ and $\partial_{t}$ are all small, this equation contains terms that are not of the same order. In fact, at leading order $\Delta F^{s}+F^{s}=0$, which selects the allowed spatial patterns and permits (3.22) to be rewritten as

$$
\partial_{t} F^{s}+\mathrm{i}(1+2 S)\left(\Delta F^{s}+F^{s}\right) / 4=-2 C_{g} F^{s}+\mathrm{i} a g(x) \bar{F}^{s} .
$$

This is the general amplitude equation, whose derivation was the main object of this subsection. Equation (3.23) applies outside four $O(1)$ layers, near the endwalls and the sidewalls, where the waves are reflected. The approximation above involves a separation of spatial scales, an assumption that does not hold in these layers. The analysis of wave reflection in appendix B (see (B 21)) provides the following boundary conditions

$$
\begin{aligned}
& \partial_{x} F^{s} \pm \int_{-1}^{1} \gamma\left(\kappa^{2}, S\right) \phi_{ \pm L_{1}}(\kappa) \mathrm{e}^{\mathrm{i} \kappa y} \mathrm{~d} \kappa=0 \quad \text { at } x= \pm L_{1}, \\
& \partial_{y} F^{s} \pm \int_{-1}^{1} \gamma\left(\nu^{2}, S\right) \phi_{ \pm L_{2}}(\nu) \mathrm{e}^{\mathrm{i} v . x} \mathrm{~d} \nu=0 \quad \text { at } y= \pm L_{2},
\end{aligned}
$$

where the real scalar $\gamma$ is as given in (B 20) (and plotted for various values of $S$ in figure 15) and the Fourier transforms $\phi_{ \pm L_{1}}$ and $\phi_{ \pm L_{2}}$ are

$$
F^{s}\left(x, \pm L_{2}\right)=\int_{-1}^{1} \phi_{ \pm I_{2}}(v) \mathrm{e}^{\mathrm{i} \nu x} \mathrm{~d} \nu, \quad F^{s}\left( \pm L_{1}, y\right)=\int_{-1}^{1} \phi_{ \pm L_{1}}(\kappa) \mathrm{e}^{\mathrm{i} \kappa y} \mathrm{~d} \kappa .
$$

Note that (3.23)-(3.25) are invariant under the reflection symmetries

$$
\left(x, F^{s}\right) \rightarrow\left(-x, \mathrm{i} F^{s}\right) \text { and } y \rightarrow-y,
$$

which follow from the symmetries (2.15) and the expansion (3.1).

Equations (3.23)-(3.25) will be used in the remainder of the paper. They are much simpler than current approximations since (3.23) is a scalar equation depending on the two horizontal coordinates only. Compared with the quasi-potential approximation, note that pressure, velocity and dependence on both the vertical coordinate $z$ and the fast time scale $t \sim 1$ are filtered out. Equation (3.23) is linear and thus excludes wave-wave interactions, which could be enhanced by triad resonances (McGoldrick 1965), particularly at very small values of $C_{g}$ (see below). Interactions with the harmonic counterpropagating wavetrains can be ignored because of the last assumption in (2.16), noting that the $\mathrm{OBF}$ and the wavetrains penetrate a distance from the endwalls that scales with $d$ and $1 / C_{g}$, respectively.

\section{Subharmonic instability and associated patterns}

\subsection{Subharmonic instability}

Since the coefficients of (3.23) do not depend on $y$, subharmonic instability can be analysed by decomposing the solution into normal modes in the $y$ direction, namely setting

$$
F^{s}=\sum_{m=0}^{\infty} F_{m}(x, t) \cos \left(\kappa_{m} y+\delta_{m}\right),
$$

where the transverse wavenumbers $\kappa_{m}$ and the phase shifts $\delta_{m}$ must be such that

$$
0 \leqslant \kappa_{m} \leqslant 1, \quad-\pi<\delta_{m}<\pi,
$$


and are determined by the equations

$$
\begin{gathered}
-\kappa_{m} \sin \left(\kappa_{m} L_{2}+\delta_{m}\right)+\gamma\left(1-\kappa_{m}^{2}, S\right) \cos \left(\kappa_{m} L_{2}+\delta_{m}\right)=0, \\
-\kappa_{m} \sin \left(-\kappa_{m} L_{2}+\delta_{m}\right)-\gamma\left(1-\kappa_{m}^{2}, S\right) \cos \left(-\kappa_{m} L_{2}+\delta_{m}\right)=0,
\end{gathered}
$$

which result from substituting (4.1) into the boundary conditions at $y= \pm L_{2}$ in (3.25). After some algebra, equations (4.3)-(4.4) yield

$$
\delta_{m}=m \pi / 2(\bmod 2 \pi), \quad \tan \left(\kappa_{m} L_{2}+\delta_{m}\right)=\gamma\left(1-\kappa_{m}^{2}, S\right) / \kappa_{m} \quad \text { with } 0<\kappa_{m} \leqslant 1 .
$$

These equations define the discrete values of the transverse wavenumber $\kappa_{m}$ that are compatible with the boundary conditions at the sidewalls and provide many values of $\kappa_{m}$ (of the order of $L_{2} / \pi$ ) because $L_{2}$ is large. These are sorted such that $0<\kappa_{1}<\kappa_{2}<\cdots$. Note from (4.1) that even and odd values of $m$ provide modes whose dependence on $y$ is even and odd, respectively, which is consistent with the $y$-reflection symmetry in (3.27).

Substitution of (4.1) into (3.23)-(3.24) leads to

$$
\begin{gathered}
\partial_{t} F_{m}+\mathrm{i}(1+2 S)\left[\partial_{x x} F_{m}+v_{m}^{2} F_{m}\right] / 4=-2 C_{g} F_{m}+\mathrm{i} a g(x) \bar{F}_{m}, \\
\partial_{x} F_{m} \pm \gamma\left(1-v_{m}^{2}, S\right) F_{m}=0 \quad \text { at } x= \pm L_{1},
\end{gathered}
$$

where

$$
v_{m}=\sqrt{1-\kappa_{m}^{2}} \geqslant 0
$$

is the longitudinal wavenumber because, since $\partial_{t}, a, C_{g} \ll 1$, at leading-order (4.6) yields $F_{m} \sim \mathrm{e}^{ \pm \mathrm{i} v_{m} x}$. The angle of the wavevector with the endwalls is thus given by

$$
\varphi=\sin ^{-1} v_{m} .
$$

Marginal instability is determined by demanding purely oscillatory solutions to the linear problem (4.6)-(4.7), setting

$$
F_{m}(x, t)=A_{m} F_{m}^{+}(x) \mathrm{e}^{\mathrm{i} \Omega t}+\bar{A}_{m} \bar{F}_{m}^{-}(x) \mathrm{e}^{-\mathrm{i} \Omega t},
$$

where the complex amplitude $A_{m}$ is arbitrary in the present linear approximation, and the eigenfunctions $F_{m}^{ \pm}$satisfy

$$
\begin{gathered}
\mathrm{i} \Omega F_{m}^{+}+\mathrm{i}(1+2 S)\left[\partial_{x x} F_{m}^{+}+v_{m}^{2} F_{m}^{+}\right] / 4=-2 C_{g} F_{m}^{+}+\mathrm{i} a g(x) F_{m}^{-}, \\
\mathrm{i} \Omega F_{m}^{-}-\mathrm{i}(1+2 S)\left[\partial_{x x} F_{m}^{-}+v_{m}^{2} F_{m}^{-}\right] / 4=-2 C_{g} F_{m}^{-}-\mathrm{i} a g(x) F_{m}^{+}, \\
\partial_{x} F_{m}^{+} \pm \gamma\left(1-v_{m}^{2}, S\right) F_{m}^{+}=\partial_{x} F_{m}^{-} \pm \gamma\left(1-v_{m}^{2}, S\right) F_{m}^{-}=0 \quad \text { at } x= \pm L_{1},
\end{gathered}
$$

as obtained by substituting (4.10) into (4.6)-(4.7). It is anticipated that

$$
|\Omega| \ll 1,
$$

which is consistent with the separation of scales assumption (3.14).

Equations (4.11)-(4.13) are invariant under the actions

$$
\left(x, F_{m}^{+}, F_{m}^{-}\right) \rightarrow\left(-x, F_{m}^{+},-F_{m}^{-}\right), \quad\left(\Omega, F_{m}^{+}, F_{m}^{-}\right) \rightarrow\left(-\Omega, \bar{F}_{m}^{-}, \bar{F}_{m}^{+}\right),
$$

which imply, in particular, that eigenvalues appear in complex conjugate pairs. Assuming simple eigenvalues (the generic case), the first symmetry in (4.15) 

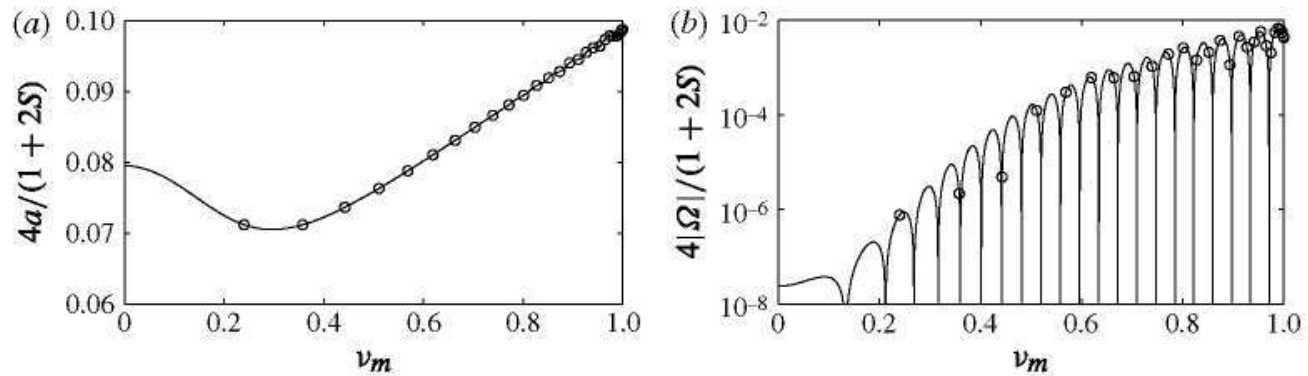

FIGURE 4. (a) Rescaled marginal instability threshold amplitude $a$ and $(b)$ rescaled frequency $\Omega$ versus the longitudinal wavenumber $v_{m}$ for $L_{1}=41.5, d=46.1, C_{g}=0.027$ and $S=0.63$. The discrete values of $v_{m}$ for $L_{2}=41.5$ are plotted with circles.

implies that

$$
F_{m}^{+}(-x) \equiv F_{m}^{+}(x), \quad F_{m}^{-}(-x) \equiv-F_{m}^{-}(x),
$$

which permits restricting to the interval $0 \leqslant x \leqslant L_{1}$. Numerical integration is performed using two methods and the results are used for mutual validation, as explained in appendix C. In particular, for fixed values of $S, C_{g}, L_{1}$ and $d$ (implicit in the function $g$, see (2.34)), a marginal instability curve is obtained,

$$
a=a\left(v_{m}\right), \quad \Omega=\Omega\left(v_{m}\right) .
$$

In fact, infinitely many such curves are obtained (with generally increasing values of a), but only that branch providing the smallest value of $a$ (which will be referred to below as the first branch) needs to be considered here; see $\$ \S 5.2$ and 5.3 below for further discussion of the remaining branches. The minimum value of $a$ on this first branch, $a_{c}$, corresponds to the instability threshold in the continuous case, as $L_{2} \rightarrow \infty$. The associated minimum when only discrete values of $v_{m}$ satisfying (4.5) and (4.8) are considered, will be denoted by $a_{c}^{d}$.

Figure 4 shows a representative plot (corresponding to one of the experimental runs considered in $\S 5$ ) of the functions (4.17) along the first branch where, for convenience, $a$ and $\Omega$ are both rescaled with $(1+2 S) / 4$, and the allowed discrete values of $v_{m}$ are indicated with circles. In this case $a_{c}^{d}=0.04$, which corresponds to $m=25$ and $v_{m}=0.26$, meaning that the pattern is antisymmetric in $y$ because $m$ is odd. Furthermore, the wave field is oblique because, according to (4.9), the wavevector forms an angle $\varphi=\sin ^{-1} 0.26=75^{\circ}$ with the endwalls. Note that (consistent with the large value of $L_{2}$ ) the discrete threshold $a_{c}^{d}$ is very close to continuous threshold, $a_{c}=0.0399$, which corresponds to $v_{m}=0.30$. As anticipated, $\Omega$ is quite small (in fact, $\Omega \sim 10^{-6}$ at threshold), which is consistent with the large value of $L_{1}$; see below.

Since $\Omega$ is small, the second symmetry (4.15) implies that eigenfunctions can always be selected (requiring that $F_{m}^{+}\left(L_{1}\right)=-\bar{F}_{m}^{-}\left(L_{1}\right)$ ) such that

$$
F_{m}^{L}=F_{m}^{+}+\bar{F}_{m}^{-} \simeq 0 \quad \text { if } x>0, \quad F_{m}^{R}=\mathrm{i}\left(F_{m}^{+}-\bar{F}_{m}^{-}\right) \simeq 0 \text { if } x<0,
$$

which means that these two functions are concentrated near the left and right endwalls, respectively. Also, equation (4.16) implies that these two functions are such that

$$
F_{m}^{L}(-x)=\mathrm{i} F_{m}^{R}(x) .
$$



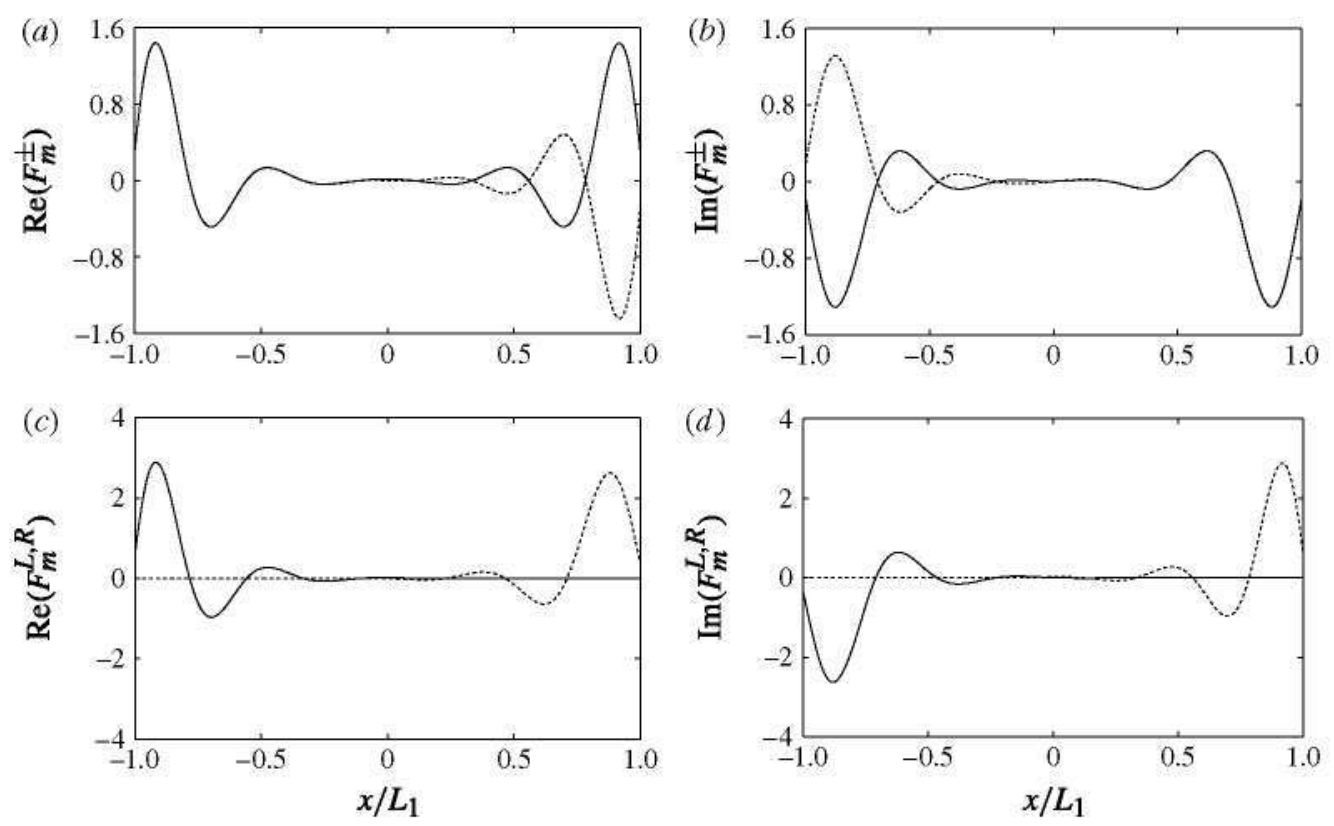

FIGURE 5. (a) Real and (b) imaginary parts of the eigenmodes $F_{m}^{+}$(solid lines) and $F_{m}^{-}$ (dashed lines) versus $x / L_{1}$, at the instability threshold for the case considered in figure $4 .(c)$ Real and $(d)$ imaginary parts of $F_{m}^{L}$ (solid lines) and $F_{m}^{R}$ (dashed lines).

To illustrate (4.18)-(4.19), the functions $F_{m}^{ \pm}, F_{m}^{L}$ and $F_{m}^{R}$ at threshold are plotted in figure 5 for the case considered in figure 4 . Note that

$$
\operatorname{Re}\left(F_{m}^{L}\right) \simeq-\operatorname{Im}\left(F_{m}^{L}\right) \text { and } \operatorname{Re}\left(F_{m}^{R}\right) \simeq \operatorname{Im}\left(F_{m}^{R}\right)
$$

this specific to this case and does not hold so clearly for other parameter values.

The reconstructed spatiotemporal patterns are obtained by substituting (4.10) into (4.1) (retaining only one mode), substituting this latter expression into (3.1) and considering only subharmonic waves. The free surface elevation is given by

$$
f=f_{m}(x, t) \cos \left(\kappa_{m} y+\delta_{m}\right),
$$

where the longitudinal part of the pattern $f_{m}$ is written as

$$
\begin{aligned}
f_{m} & =\mathrm{e}^{\mathrm{i} t}|A|\left[\mathrm{e}^{\mathrm{i} \psi(t)} F_{m}^{+}+\mathrm{e}^{-\mathrm{i} \psi(t)} \bar{F}_{m}^{-}\right]+\text {c.c. } \\
& \equiv \mathrm{e}^{\mathrm{i} t}|A|\left[F_{m}^{L} \cos \psi(t)+F_{m}^{R} \sin \psi(t)\right]+\text { c.c. },
\end{aligned}
$$

with $F_{m}^{L, R}$ as defined in (4.18) and the slowly varying phase $\psi$ defined as

$$
\psi=\Omega t+\text { phase of } A(\bmod 2 \pi) .
$$

This represents a quasi-periodic pattern that exhibits the frequencies 1 and $\Omega \ll 1$.

Since $F_{m}^{L}$ and $F_{m}^{R}$ are concentrated near the left and right endwalls (see figure 5), four distinguished fast time behaviours in the interval $0 \leqslant t \leqslant \pi$ occur depending on $\psi$. These behaviours are identified noting that activity is concentrated near the left and right endwalls for: (i) $\psi(t)=0$; and (iii) $\psi=\pi / 2$, respectively, and present near both endwalls for: (ii) $\psi(t)=\pi / 4$; and (iv) $\psi(t)=3 \pi / 4$. Thus, as $\psi(t)$ increases on the slow time scale, the system cycles through the fast time behaviours (i), (ii), (iii), (iv), 

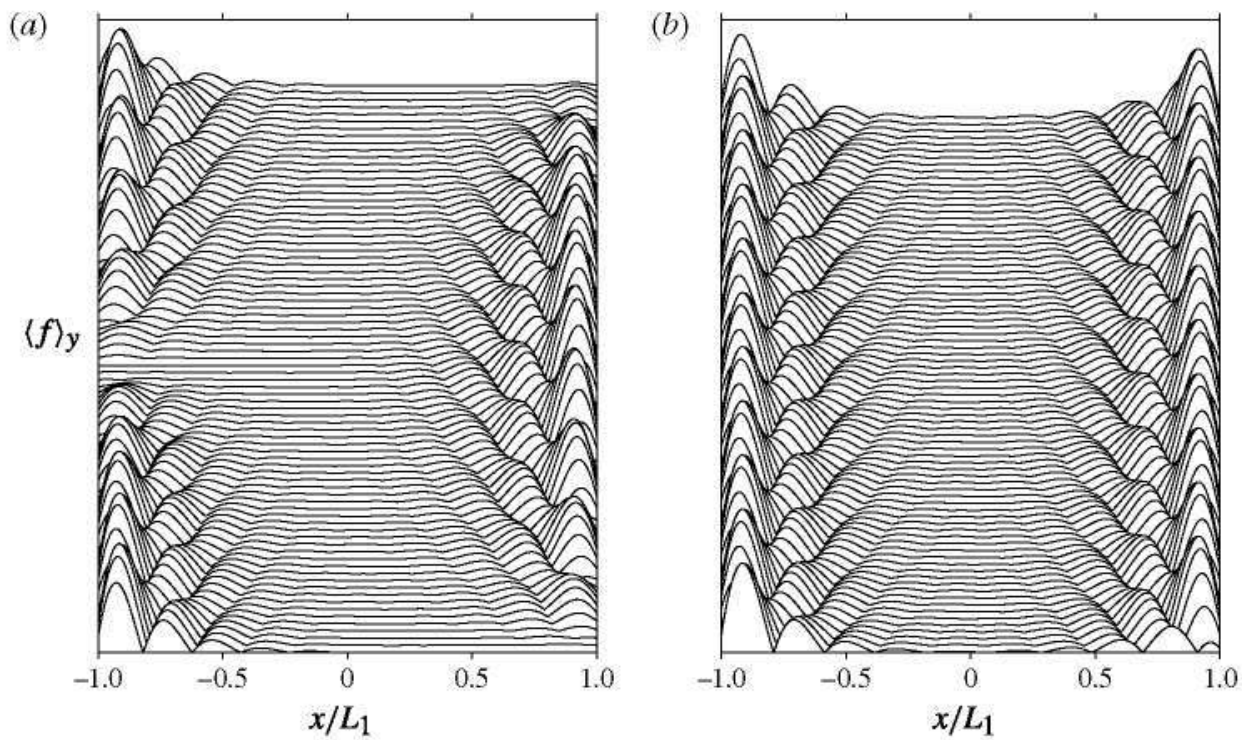

FIGURE 6. Space-time plots of the transverse root mean square of the free surface elevation, $\langle f\rangle_{y}$, defined in (4.25) of type I $(a)$ and type II $(b)$ patterns with $\Omega=0.1$ (much larger than typical experimental values to reduce the time interval) and $L_{2}=50$.

(i), (ii), ..., and so on. This can be seen in figure $6(a)$, where a space-time plot of the transverse root mean square of the free surface elevation,

$$
\langle f\rangle_{y}=\sqrt{\frac{1}{2 L_{2}} \int_{-L_{2}}^{L_{2}} f^{2} \mathrm{~d} y},
$$

is given. Note that $\langle f\rangle_{y} \sim f_{m}$ for the threshold patterns.

\subsection{Primary and secondary spatiotemporal patterns}

In the formulation above, the subharmonic instability is associated with a Hopf bifurcation of the flat solution $F^{s}=0$. In terms of the original formulation (2.4)-(2.6), (2.10)-(2.14), a system with periodic coefficients, the bifurcation is associated with a pair of complex Floquet exponents, $\mathrm{i}(\pi \pm \Omega$ ) (whose Floquet multipliers are both close to -1). Both approaches are equivalent. Here we consider the Hopf bifurcation, which is analysed by adding cubic nonlinear terms in the general amplitude equation (3.23) and performing a weakly nonlinear analysis (Guckenheimer \& Holmes 1983; Kuznetsov 1998). The complex amplitude $A$ of the marginal mode satisfies a Landau equation,

$$
A^{\prime}(t)=\beta_{1}\left(a-a_{c}\right) A-\beta_{2}|A|^{2} A,
$$

where the real part of $\beta_{1}$ is positive (because the instability sets in for $a>a_{c}$ ) and the real part of $\beta_{2}$ is also positive if the bifurcation is supercritical, as we assume hereafter. As time increases, $A(t)$ converges to a periodic solution of the form $A=A^{0} \mathrm{e}^{\mathrm{i} \Omega_{1^{t}}}$, where $\left|A^{0}\right|^{2} \sim\left|\Omega_{1}\right| \ll\left|a-a_{c}\right|$ as $a \rightarrow a_{c}$. Substituting this into (4.10) just provides a small correction of the frequency $\Omega$ in the temporally modulated patterns described in the last subsection, which are the expected patterns when $\left|a-a_{c}\right|$ is sufficiently small and the bifurcation is supercritical. 
On the other hand, since the transverse length $L_{2}$ is large, interaction with nearby modes is expected near threshold. Because the instability thresholds of the interacting modes are near the minimum of the curve $a=a\left(\kappa_{m}\right)$ defined in (4.17), (4.3)-(4.4), (4.8), (4.11)-(4.13) show that

$$
\begin{aligned}
\left|\kappa_{m}-\kappa_{m-1}\right| & \sim\left|v_{m}-v_{m-1}\right| \sim\left|F_{m}^{ \pm}-F_{m-1}^{ \pm}\right| \\
& \sim\left|\Omega_{m}-\Omega_{m-1}\right| \sim \sqrt{\left|a_{c, m}-a_{c, m-1}\right|} \sim 1 / L_{2}^{2} \ll 1 .
\end{aligned}
$$

This is clear in figure $4(a)$, where the mode $m=25$ bifurcates first, but the $m-1=24$ mode is nearly marginal. Interaction between the $(m-1)$ th and $m$ th modes is accounted for by considering the free surface pattern

$$
\begin{aligned}
f= & \mathrm{e}^{\mathrm{i} t}\left[A_{m-1} F_{m-1}^{+} \mathrm{e}^{\mathrm{i} \Omega t}+\bar{A}_{m-1} \bar{F}_{m-1}^{-} \mathrm{e}^{-\mathrm{i} \Omega t}\right] \sin \left(\kappa_{m-1} y+m \pi / 2\right) \\
& +\mathrm{e}^{\mathrm{i} t}\left[A_{m} F_{m}^{+} \mathrm{e}^{\mathrm{i} \Omega t}+\bar{A}_{m} \bar{F}_{m}^{-} \mathrm{e}^{-\mathrm{i} \Omega t}\right] \cos \left(\kappa_{m} y+m \pi / 2\right)+\text { c.c. }+\cdots,
\end{aligned}
$$

where the coupled evolution of $A_{m-1}$ and $A_{m}$ is given by the amplitude equations

$$
\begin{aligned}
A_{m-1}^{\prime} & \simeq\left[\beta_{1}\left(\varepsilon-\delta_{1}\right)-\mathrm{i} \delta_{2}\right] A_{m-1}-\left(\beta_{2}\left|A_{m-1}\right|^{2}+\beta_{3}\left|A_{m}\right|^{2}\right) A_{m-1}+\beta_{4} \bar{A}_{m-1} A_{m}^{2}, \\
A_{m}^{\prime} & \simeq\left[\beta_{1}\left(\varepsilon+\delta_{1}\right)+\mathrm{i} \delta_{2}\right] A_{m}-\left(\beta_{2}\left|A_{m}\right|^{2}+\beta_{3}\left|A_{m-1}\right|^{2}\right) A_{m}+\beta_{4} \bar{A}_{m} A_{m-1}^{2} .
\end{aligned}
$$

These equations correspond to a double Hopf bifurcation with strong 1:1 resonance (Guckenheimer \& Holmes 1983; Kuznetsov 1998), with the additional requirement that they are invariant under the actions $A_{m-1} \rightarrow-A_{m-1}$ and $A_{m-1} \leftrightarrow A_{m}$, which are associated with the transformations $y \rightarrow-\left(y+m \pi / \kappa_{m}\right)$ and $y \rightarrow \pi /\left(2 \kappa_{m}\right)-y$, respectively. The slow frequency $\Omega$ appearing in (4.28), the bifurcation parameter, the threshold splitting $\delta_{1}$ and the detuning $\delta_{2}$ in (4.29)-(4.30) are defined in terms of the threshold amplitudes and eigenfrequencies of the two interacting modes as

$$
\left.\begin{array}{ll}
\Omega=\left(\Omega_{m-1}+\Omega_{m}\right) / 2, & \varepsilon=a-\left(a_{c, m-1}+a_{c m}\right) / 2, \\
\delta_{1}=\left(a_{c, m-1}-a_{c, m}\right) / 2, & \delta_{2}=\left(\Omega_{m-1}-\Omega_{m}\right) / 2 .
\end{array}\right\}
$$

Equations (4.29)-(4.30) exhibit pure mode solutions, $\left(A_{m-1}, 0\right)$ and $\left(0, A_{m}\right)$. For these, equations (4.29)-(4.30) decouple and reduce to the Landau equation (4.26) considered above; pure modes arise in the primary bifurcations at $a=a_{c, m-1}$ and $a=a_{c, m}$. There are also mixed mode solutions, with $A_{m-1} \neq 0$ and $A_{m} \neq 0$, that generally provide more complex temporally modulated patterns. In particular, if

$$
\left|\delta_{1}\right|,\left|\delta_{2}\right| \ll \varepsilon,
$$

then there are two types of simple mixed modes,

$$
\text { type I: } \quad\left(A_{m-1}, A_{m}\right) \simeq( \pm A, A), \quad \text { type II: } \quad\left(A_{m-1}, A_{m}\right) \simeq( \pm \mathrm{i} A, A),
$$

where the + and - signs correspond to reflection in $y$ and provide similar patterns.

Type I modes produce patterns that are approximated by substituting $\left(A_{m-1}, A_{m}\right)=$ (A,A) into (4.28) then setting $\kappa_{m-1}=\kappa_{m}$ and $F_{m-1}^{ \pm}=F_{m}^{ \pm}$(see (4.27)) to obtain

$$
f=\sqrt{2} \mathrm{e}^{\mathrm{i} t}|A|\left[F_{m}^{L} \cos \psi(t)+F_{m}^{R} \sin \psi(t)\right] \sin \left(\kappa_{m} y+m \pi / 2+\pi / 4\right)+\text { c.c. },
$$

where $F_{m}^{L, R}$ are as defined in (4.18) and the slowly varying phase $\psi$ is given by (4.24). Comparison with (4.21)-(4.24) shows that this pattern is quite similar to the primary pattern (except for the factor $\sqrt{2}$ and the $\pi / 4$ phase shift in the $y$ direction). Figure 6(a) shows that wave activity alternates between the endwalls on the slow time scale. 

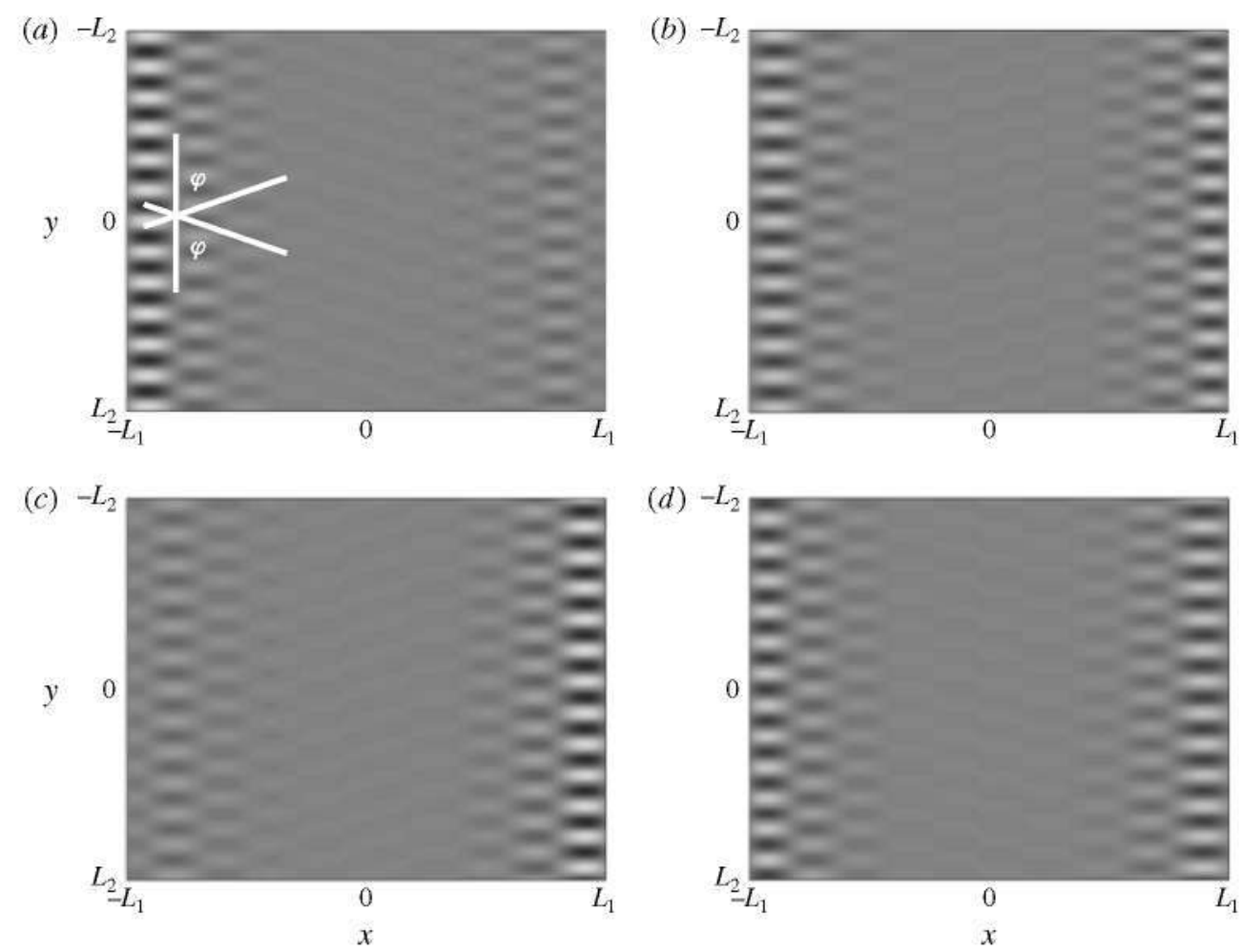

FIGURE 7. Greyscale contours of the free surface elevation (as given by (4.35) with $\psi(t)=\pi / 4)$ at $(a) t=\pi / 4,(b) t=\pi / 2,(c) t=3 \pi / 4$ and $(d) t=\pi$, for the case in figures 4-5, considering the interaction of the $m-1=24$ th and $m=25$ th modes.

The patterns associated with mixed modes of type II, instead, are different from the primary patterns. Substituting $\left(A_{m-1}, A_{m}\right)=(\mathrm{i} A, A)$ into $(4.28)$ and setting $\kappa_{m-1}=\kappa_{m}$ and $F_{m-1}^{ \pm}=F_{m}^{ \pm}$, as above, lead to

$$
f=\mathrm{e}^{\mathrm{i} t}|A|\left[F_{m}^{L} \cos \left[\kappa_{m} y+m \pi / 2+\psi(t)\right]+F_{m}^{R} \sin \left[\kappa_{m} y+m \pi / 2+\psi(t)\right]\right]+\text { c.c. }
$$

Thus, these patterns show similar activity near both endwalls, as seen in figure $6(b)$. The effect of the slow frequency does not lead here to any left-right modulation of the pattern; instead, it just induces a slow drift in the transverse direction. Invoking (4.19), this pattern is invariant on the fast time scale under the spatiotemporal symmetry

$$
t \rightarrow t+\pi / 2, \quad x \rightarrow-x, \quad y \rightarrow(2 \psi-m \pi+\pi / 2) / \kappa_{m}-y,
$$

consistent with the basic symmetry (3.27). This symmetry and the obliqueness of the pattern are evident in figure 7 , where four representative snapshots of one period on the fast scale are considered. The first and third snapshots also show that the wave intensity is fairly small near the right and left endwalls, respectively, but this is due to the property (4.20), which is specific to the particular case that is being considered; for other parameter values, the fast left-right shift in wave intensity is less pronounced.

The small difference between $\left(F_{m-1}^{ \pm}, \kappa_{m-1}\right)$ and $\left(F_{m}^{ \pm}, \kappa_{m}\right)$, not accounted for above, produces small differences in longitudinal wavelength near both endwalls and, more importantly, a transverse modulation. This could be accounted for by using the full 
expression (4.28) to calculate the patterns (4.34) and (4.35), which would show that the amplitude of the pattern varies by an $O(1)$ quantity in the transverse direction. This transverse modulation is due to the interaction of just two modes, which locally cancel or reinforce each other. This effect is very sensitive to the wavelengths, amplitudes and phases of the interacting modes. In other words, transverse modulations are very much affected by the neglected differences between the coefficients in the amplitude equations (4.29)-(4.30) and the symmetry-breaking parameters $\delta_{1}$ and $\delta_{2}$. Analysis of these is well beyond the scope of this paper.

We can summarize the above as follows.

(i) The patterns are generally oblique.

(ii) The primary patterns at threshold are quasi-periodic, exhibit two well-separated time scales and show activity that cycles slowly between both endwalls; see figure $6(a)$.

(iii) Since $L_{2} \gg 1$, interaction with modes exhibiting nearby wavenumbers is expected very soon after threshold and produces two types of mixed-mode patterns. Type I patterns are qualitatively similar to the primary patterns. Type II patterns, instead, show similar activity near both the two endwalls (see figure $6 b$ ). Both types of mixed-mode patterns may also show a significant transverse modulation, but this is affected by several small effects that have been neglected above.

(iv) Since the second frequency $\Omega$ is small, experimental observation of the slow time scale may require very long experimental runs. In shorter runs, the portion of the slow oscillation of type I patterns that is visualized depends on initial conditions, and thus may change (in an apparently random fashion) from one run to another.

(v) More complex modulated patterns resulting from, e.g., time-dependent solutions of (4.29)-(4.30), interaction between three or more modes and cases in which the two bifurcations mentioned above are subcritical are to be expected as $a-a_{c}$ increases further, but all of these are again beyond the scope of this paper.

\section{Results}

The theory developed in the last section is used here to both: (i) calculate specific results that are compared with their experimental counterparts (in \$5.1); and (ii) elucidate the dependence of both the instability threshold and the resulting primary patterns on the various non-dimensional parameters (in $\$ \S 5.2-5.4$ ). The validity of the theory relies on the assumptions (2.16), which in practice require that $2 L_{1} \gtrsim 12 \pi$, $2 L_{2} \gtrsim 12 \pi$ (for at least six wavelengths to be present in the container), $\mathrm{e}^{-d} \ll 1$ and $C_{g} \ll 1$. These can be generally considered to hold provided that

$$
L_{1}, L_{2} \gtrsim 15, \quad d \gtrsim 5 \text { and } C_{g} \lesssim 0.1
$$

\subsection{Comparison with experiments}

The non-dimensionalization used in this paper is very convenient for theoretical purposes. To compare with experiments, dimensions are restored by solving the cubic equation (2.2) at each $\omega^{*}$ (=one half of the forcing frequency), which is varied in experimental runs. Then, the dimensional threshold amplitude $a^{*}=k^{*} a_{c}$, the angle $\varphi$ defined in (4.9) that provides the obliqueness of the patterns, and the dimensional frequency associated with the slow modulations $\Omega^{*}=\Omega / \omega^{*}$ are readily calculated.

Comparison is made in figure 8 with the experiments by Porter et al. (2012), performed in a rectangular container of depth $d^{*}=5 \mathrm{~cm}$ and cross-section $2 L_{1}^{*} \times 2 L_{2}^{*}=$ $9 \mathrm{~cm} \times 9 \mathrm{~cm}$, using two silicone oils, with viscosities $\mu=5$ and $10 \mathrm{cSt}$ over 


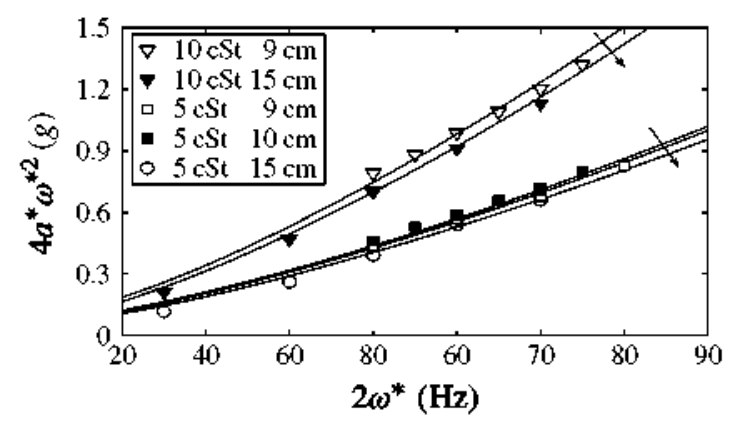

FIGURE 8. The dimensional threshold acceleration $4 a^{*} \omega^{* 2}$ versus the forcing frequency $2 \omega^{*}$ resulting from several experimental runs (symbols) and from the theory developed in the last section for $\mu=5 \mathrm{cSt}$ (lower curves) and $10 \mathrm{cSt}$ (upper curves); arrows indicate increasing cross-section.

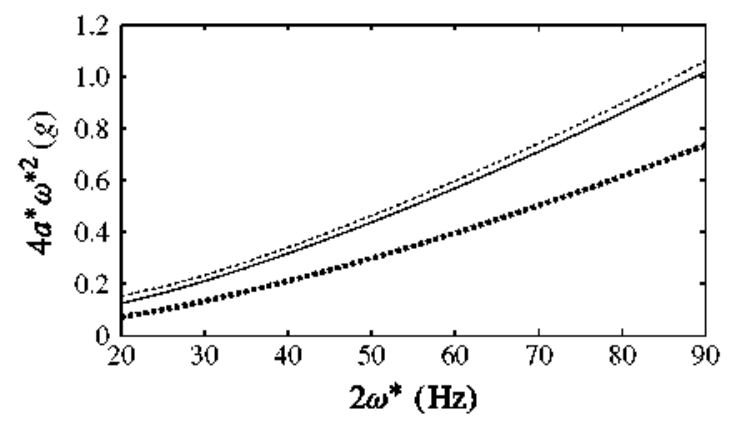

FIGURE 9. Comparison of the threshold acceleration calculated above (solid line) with that obtained using Dirichlet (thin dashed line) and Neumann (thick dashed line) boundary conditions for the case of $5 \mathrm{cSt}$ silicone oil in a $9 \mathrm{~cm} \times 9 \mathrm{~cm}$ container, in figure 8 .

the frequency range $25-80 \mathrm{~Hz}$. For the less-viscous fluid $\sigma \sim 19.7 \mathrm{dyn} \mathrm{cm}^{-1}$ and $\rho=0.913 \mathrm{~g} \mathrm{~cm}^{-3}$, while for the other $\sigma=20.1 \mathrm{dyn} \mathrm{cm}^{-1}$ and $\rho=0.933 \mathrm{~g} \mathrm{~cm}^{-3}$. The non-dimensional parameters defined in this paper vary over the ranges $25 \leqslant L_{1} \leqslant$ $100,25 \leqslant L_{2} \leqslant 100,30 \leqslant d \leqslant 70,0.01 \leqslant C_{g} \leqslant 0.08$ and $0.2 \leqslant S \leqslant 0.8$, which can be considered to satisfy the assumptions (2.16). Care was taken to maintain the contact line fixed, as assumed above, but slight motions were sometimes unavoidable. In order to further check the theoretical results above, an additional set of experiments have been performed using the same experimental procedure and the same silicone oils, but in containers with cross-sections of $2 L_{1}^{*} \times 2 L_{2}^{*}=10 \mathrm{~cm} \times 10 \mathrm{~cm}$ and $15 \mathrm{~cm} \times 15 \mathrm{~cm}$. Note in figure 8 that the theory agrees with the experiments very well; in fact, the theory predicts the threshold acceleration within the experimental uncertainty, which is $\sim 5 \%$.

We remark that using the appropriate boundary conditions at the sidewalls and endwalls (namely, (4.13)) is essential quantitatively, as illustrated in figure 9, where one of the instability curves in figure 8 is compared with its counterparts using Dirichlet and Neumann boundary conditions,

$$
F_{m}^{ \pm}=0 \quad \text { and } \quad\left(\partial_{x} F_{m}^{ \pm}, \partial_{y} F_{m}^{ \pm}\right)=(0,0) \quad \text { at }(x, y)=\left( \pm L_{1}, \pm L_{2}\right)
$$



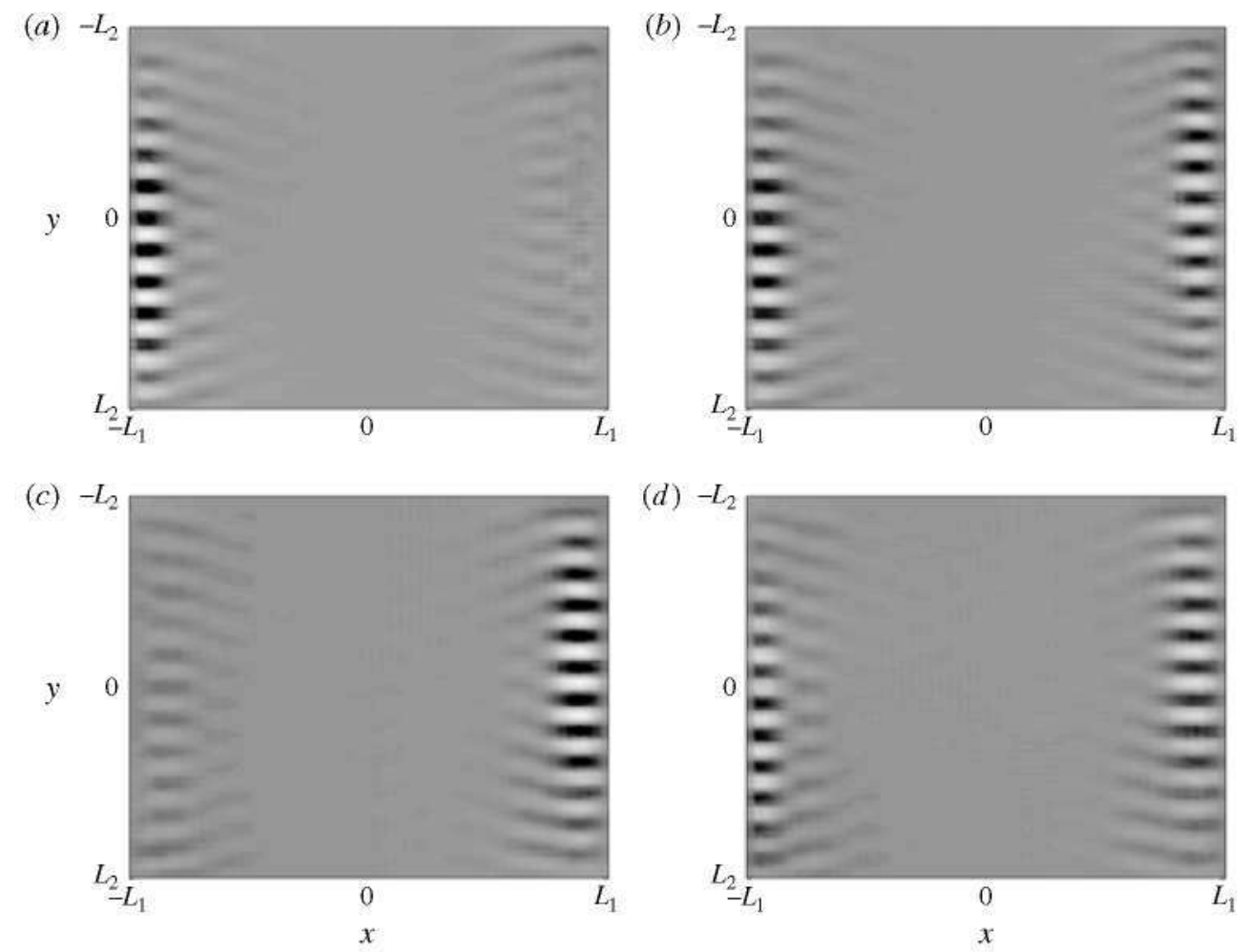

FIGURE 10. Counterpart of figure 7 for the experimental run corresponding to the non-dimensional parameter values considered in that figure.

respectively. Using Neumann boundary conditions leads to a $30 \%$ error. The difference with the Dirichlet case is smaller because the constant $\gamma$ appearing in (4.13) is somewhat large ( $\gamma \simeq 2$, see figure 15 ). Clearly, the instability threshold is very much affected by the way in which the contact line is treated, either fixed (as done in this paper) or allowed to move, and this is a source of experimental uncertainty, as indicated above.

The theoretically computed values of the pattern angle $\varphi$ and the modulation frequency are in the ranges $71^{\circ} \leqslant \varphi \leqslant 76^{\circ}$ and $10^{-10} \leqslant \Omega \leqslant 10^{-2}$, respectively, which is consistent with the measurements by Porter et al. (2012). A more precise comparison with these experiments is difficult because the patterns only exhibit a few wavelengths in the longitudinal direction and experimental uncertainties together with practical time constraints prevent measuring very small values of $\Omega$. Also, the precision with which the instability threshold can be approached is limited, meaning that the slowly modulated patterns illustrated in figure 6 cannot easily be identified. The patterns resulting from type I and II mixed modes, illustrated in figure 7, instead, occur for not so small $\left|a-a_{c}\right|$ and are consistent with some of the observed experimental patterns.

As an example, figure 10 shows an experimental pattern that is seen for $\mu=5 \mathrm{cSt}$, $d^{*}=5 \mathrm{~cm}$ and $2 \omega^{*}=50 \mathrm{~Hz}$. These parameters give $L_{1}=L_{2}=41.5, d=46.1$, $C_{g}=0.027$ and $S=0.63$, which is precisely the case considered for illustration in figures $4-7$. As can be seen, the experimental pattern compares fairly well qualitatively 
with its theoretical counterpart in figure 7, except for the transverse modulation, which was not accounted for in figure 7 for the reasons given. The data used to produce this image reveals that the transverse modulation in figure 10 is poorly resolved near the sidewalls and the endwalls. This is partly due to the finite precision of the synthetic Schlieren technique (Moisy, Rabaud \& Slasac 2009) and the associated particle image velocimetry (PIV) processing, and partly due to reflections off the walls of the container. As mentioned, there are also some unavoidable (slight) motions of the contact line that can have a significant effect on the boundary conditions and thus on the pattern itself. Many other experimental patterns are similar to this one (and also show a good qualitative comparison with theory), while others exhibit a more complex structure that could result, for example, either from subcritical bifurcations or from interaction of more than two nearby modes. All of them are oblique, with an angle similar to that in figure 7, consistent with the theoretically calculated angles.

Summarizing, the theoretical instability threshold compares quite well quantitatively with the experimental measurements, while the predicted patterns compare well qualitatively with the experimental visualizations (to the extent that comparison is possible).

\subsection{Marginal instability curves $a=a\left(v_{m}\right)$}

The dependence of the marginal instability curves on the various non-dimensional parameters is now examined. The marginal curves associated with the first branch (4.17) are considered first because they generally provide the smallest value of $a$. Dependence of the first branch on $L_{1}, S$ and $C_{g}$ for $d=5$ is summarized in figure 11; for other values of $d$, the marginal curves are qualitatively similar. Note that the stability threshold increases monotonically with $C_{g}$ (as viscosity is increased), which is as expected. The marginal curves are fairly smooth for the larger values of $C_{g}$, but oscillate visibly, suggesting resonance tongues for small values of $C_{g}$. These tongues peak at critical values of $v_{m}$ that are fairly constant as $C_{g} \rightarrow 0$. Also, the oscillations are steeper as $L_{1}$ increases. This behaviour for very small $C_{g}$ is analysed in the next subsection. Typically, $0<v_{m}^{c}<1$, which corresponds to strictly oblique patterns. The modulation frequency for the cases considered in figure 11 is provided in figure 12, which shows that $|\Omega|$ reaches a (relatively broad) maximum at the cusps of the tongues and that $|\Omega|$ decreases as either $C_{g}$ or $L_{1}$ increases. In fact, $\Omega \rightarrow 0$ exponentially as $L_{1} C_{g} \rightarrow \infty$.

Although the primary instability is associated with the first branch of eigenvalues of (4.11)-(4.13), the second branch is close by, especially for very small $C_{g}$. Thus, the second branch must be taken into account during numerical continuation to avoid jumps between branches. For illustration, figure 13 shows the first three branches. Note that the second and third branches break into isolas in some regions, which makes numerical continuation fairly demanding. In order to avoid the tongues being so close together and the isolas being so narrow that they cannot be seen in the plot, $L_{1}=10$ has been selected for figure 13 despite being slightly outside the parameter range considered above (cf. (5.1)).

\subsection{Spatial resonances at very small viscosity}

Even though the limit of very small $C_{g}$ is beyond the scope of this paper for the reasons given, it is useful to understand the resonance tongues appearing in figures 11 and 13, and to explain the essential differences with Faraday waves in analytical terms.

As $C_{g} \rightarrow 0$, the eigenvalue problem (4.11)-(4.13) exhibits spatial resonances when the unforced, strictly inviscid problem obtained setting $a=C_{g}=0$ in (4.11)-(4.13) has 

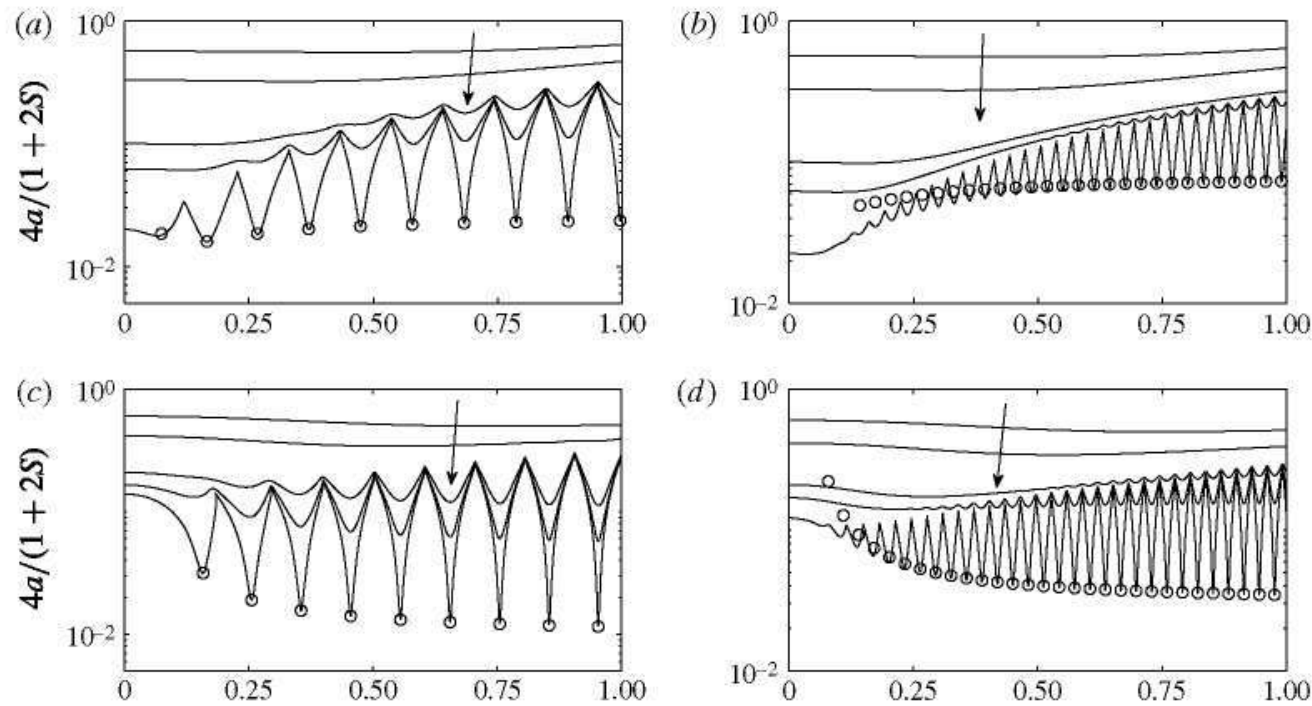

(d)
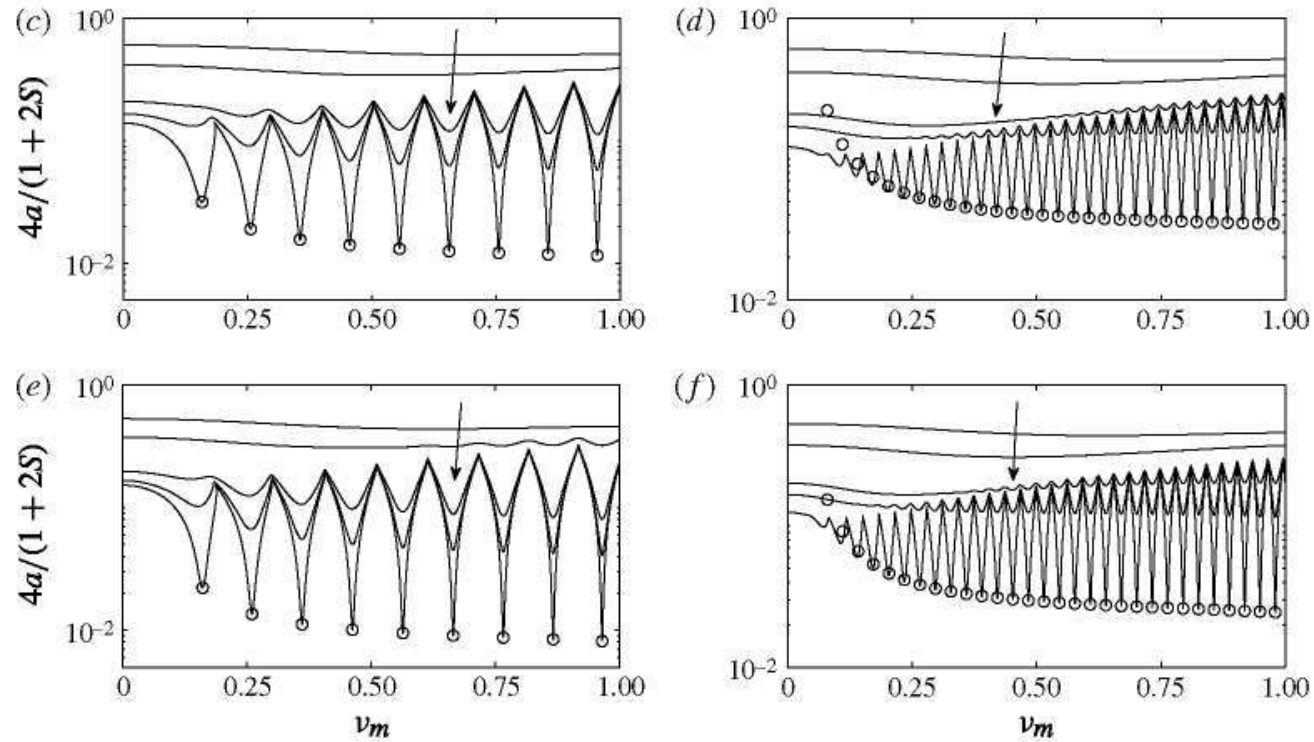

FIgURE 11. Marginal instability curves, $a_{c}$ versus $v_{m}$, for $d=5$ for $(a, c, e) L_{1}=15$ and $(b, d, f) L_{1}=50$, with $(a, b) S=0,(c, d) S=0.5$ and $(e, f) S=1$. Each plot contains curves for $C_{g}=0.001,0.005,0.01,0.05$ and 0.1 , with arrows indicating decreasing values of $C_{g}$. Circles indicate the approximate values of the minima of the curves calculated in $\$ 5.3$.

non-trivial solutions. The resulting (4.11)-(4.12) are readily solved to obtain

$$
F_{m}^{ \pm}=A^{ \pm} \cos \left(\tilde{v}_{m}^{ \pm} x+\delta_{m}^{ \pm}\right) \quad \text { with } \tilde{v}_{m}^{ \pm}=\sqrt{v_{m}^{2} \pm 4 \Omega /(1+2 S)}
$$

Here, $A^{ \pm}$are arbitrary constants, which will be related among each other below. Imposing the boundary conditions (4.13) on $F_{m}^{+}$and $F_{m}^{-}$yields (cf. (4.5))

$$
\delta_{m}^{ \pm}=0 \text { or } \pi / 2, \quad \tan \left(\tilde{v}_{m}^{ \pm} L_{1}+\delta_{m}^{ \pm}\right)=\gamma\left(1-v_{m}^{2}, S\right) / \tilde{v}_{m}^{ \pm} .
$$

These two equations (for \pm ) must hold simultaneously and determine a set of discrete values of $\left(v_{m}, \Omega\right)$; in principle, there are four combinations of the set of equations, for $\left(\delta_{m}^{-}, \delta_{m}^{+}\right)=(0,0),(0, \pi / 2),(\pi / 2,0)$ and $(\pi / 2, \pi / 2)$. As $L_{1} \rightarrow \infty, \Omega \rightarrow 0$ and $\tilde{v}_{m}^{-}$and $\tilde{v}_{m}^{+}$must be close to each other. Note that $F_{m}^{ \pm}$is an even function of $x$ for $\delta_{m}^{ \pm}=0$ and odd for $\pi / 2$.

Now, the effect of both parametric forcing and viscous damping is accounted for by adding higher-order terms in (5.3) and writing

$$
F_{m}^{ \pm}=A^{ \pm} \cos \left(\tilde{v}_{m}^{ \pm} x+\delta_{m}^{ \pm}\right)+\hat{F}_{1}^{ \pm}+\cdots
$$



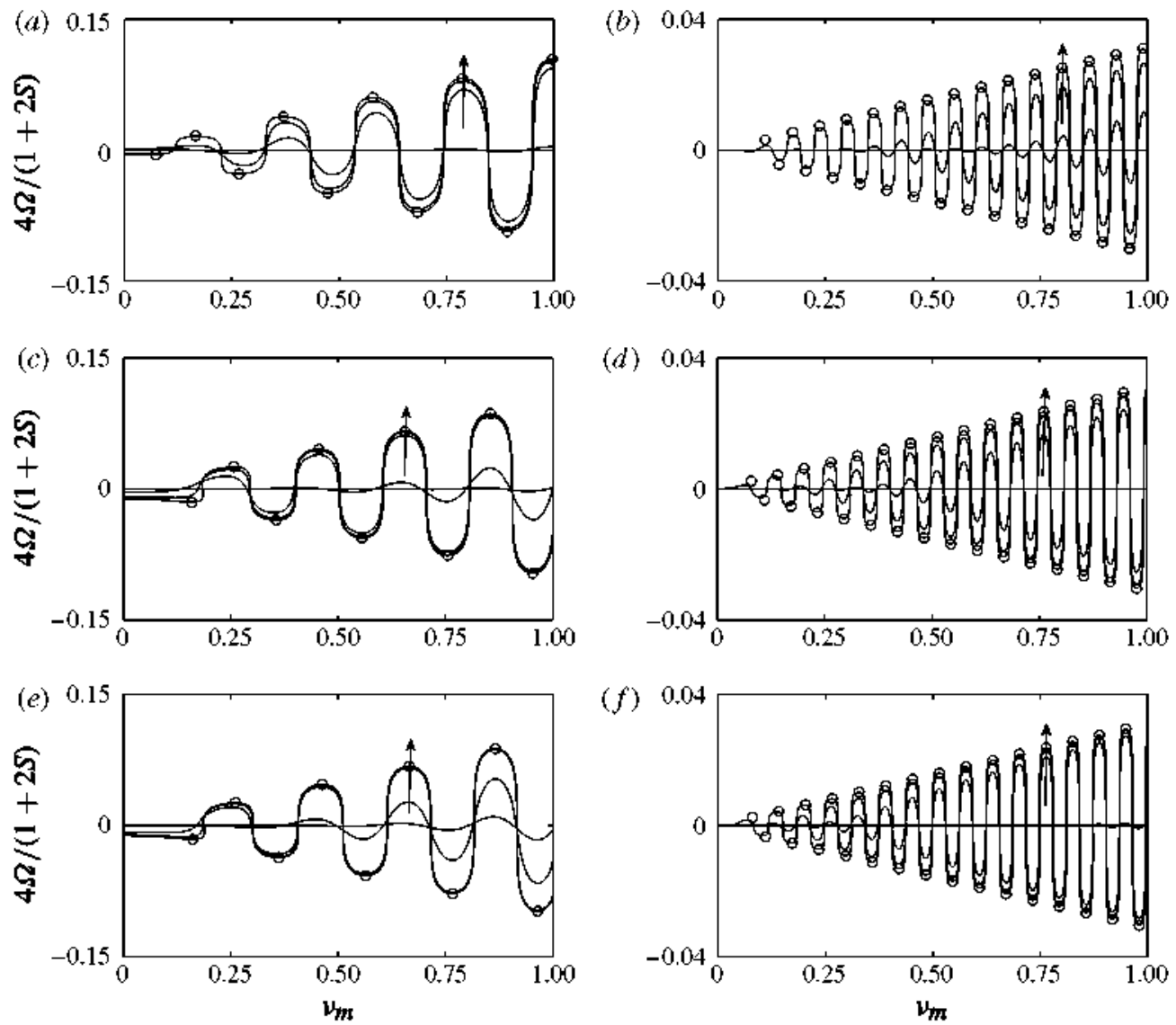

FIGURE 12. Modulation frequency $\Omega$ for the cases considered in figure 11. Circles indicate the approximate values of $\Omega$ at the minima of the curves in figure 11 , as calculated in $\$ 5.3$.

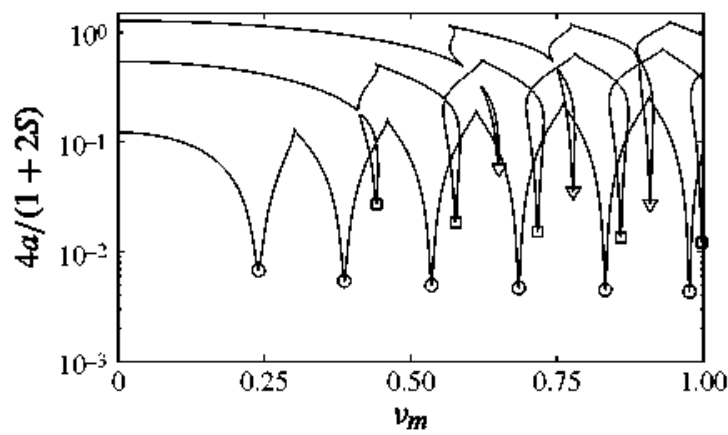

FIgURE 13. Counterpart of figure 11 for the case $d=L_{1}=10, S=1$ and $C_{8}=0.001$, considering the first three branches (solid lines) and the approximations of the minima of these curves calculated as explained in $\$ 5.3$ for the first (circles), second (squares) and third (triangles) branches. 
where $\hat{F}_{1}^{ \pm}$accounts for the leading-order corrections in the limit $C_{g}, a \rightarrow 0$. Substituting these into (4.11)-(4.13) yields

$$
\begin{gathered}
\pm \mathrm{i}(1+2 S)\left[\partial_{x x} \hat{F}_{1}^{ \pm}+\left(\tilde{v}_{m}^{ \pm}\right)^{2} F_{m}^{+}\right] / 4=-\left(\mathrm{i} \Omega_{1} \pm \hat{\delta}+2 C_{g}\right) F_{m}^{ \pm}+\mathrm{i} a g(x) F_{m}^{\mp}, \\
\partial_{x} \hat{F}_{1}^{+} \pm \gamma \hat{F}_{1}^{+}=\partial_{x} \hat{F}_{1}^{-} \pm \gamma \hat{F}_{1}^{-}=0 \quad \text { at } x= \pm L_{1},
\end{gathered}
$$

where additional terms proportional to $\gamma^{\prime} F^{ \pm}\left( \pm L_{1}\right)$ have been omitted in (5.7) because $\left|\gamma^{\prime}\right|$ and $\left|F^{ \pm}\left( \pm L_{1}\right)\right|$ are both small. Here $\Omega_{1}$ is a correction to the frequency $\Omega$ and $\hat{\delta}$ is a rescaled spatial detuning measuring the distance from the vertices of the tongues. Applying solvability conditions to the (singular) linear problem (5.6)-(5.7), a set of two linear equations in the complex amplitudes results,

$$
\tilde{\beta}_{0}^{ \pm}\left(\mathrm{i} \Omega_{1} \pm \mathrm{i} \hat{\delta}+2 C_{g}\right) A^{ \pm}= \pm \mathrm{i} a \beta_{0} A^{\mp}
$$

where

$$
\tilde{\beta}_{0}^{ \pm}=\int_{-L_{1}}^{L_{1}} \cos ^{2}\left(\tilde{v}_{m}^{ \pm} x+\delta_{m}^{ \pm}\right) \mathrm{d} x, \quad \beta_{0}=\int_{-L_{1}}^{L_{1}} g(x) \cos \left(\tilde{v}_{m}^{+} x+\delta_{m}^{+}\right) \cos \left(\tilde{v}_{m}^{-} x+\delta_{m}^{-}\right) \mathrm{d} x .
$$

Since the forcing function $g$ is odd, $\beta_{0}$ is non-zero only if the two cosines appearing in (5.9) have different parities, namely if either $\left(\delta^{+}, \delta^{-}\right)=(0, \pi / 2)$ or $(\pi / 2,0)$; both possibilities lead to the same solutions because (5.3) is invariant under the action $\left(\Omega, F_{m}^{ \pm}\right) \rightarrow\left(-\Omega, F_{m}^{\mp}\right)$. For consistency with the analysis in $\S 4.1$, we select

$$
\delta_{m}^{+}=0, \quad \delta_{m}^{-}=\pi / 2 .
$$

The associated solutions of the system (5.4) provide the values of $v_{m}$ at the vertices of the tongues in figure 11 and the corresponding values of $\Omega$. These are plotted with circles in figure 12, where it can be seen that the approximation is quite good. The threshold amplitude is calculated by requiring that the homogenous linear system (5.8) exhibits non-zero solutions, which yields $a=\sqrt{\left(4 C_{g}^{2}+\hat{\delta}^{2}\right) / \tilde{\beta}_{0}^{+} \tilde{\beta}_{0}^{-}}$and $\Omega_{1}=0$. This curve provides the marginal instability curves near each of the minima of the tongues in figure 11 . The latter are attained at $\hat{\delta}=0$, which yields

$$
a_{c}=2 C_{g} / \sqrt{\tilde{\beta}_{0}^{+} \tilde{\beta}_{0}^{-}} .
$$

These values are plotted with circles in figure 11, where it can be seen that the approximation is quite good, except for small $v_{m}$, where the asymptotic theory fails. Note that $\Omega_{1}=0$, meaning that the forcing frequency behaves as $\Omega=\Omega_{0}+O\left(\hat{\delta}^{4}\right)$ near the minima of the tongues, which is clearly observed in the flat behaviour near the minima in figure 12 .

The above is concerned with interaction between natural modes exhibiting neighbouring values of $v_{m}^{+}$and $v_{m}^{-}$. Interaction between natural modes associated with $v_{m}^{ \pm}$and $v_{m-1}^{ \pm}$and with $v_{m}^{ \pm}$and $v_{m-2}^{ \pm}$provide the minima of the marginal instability curves in the second and third branches, and are plotted in figure 13 with squares and triangles, respectively. Note that the approximation is again quite good.

The analysis above also shows that $\Omega \neq 0$ generically, because if $\Omega=0$, then $F_{m}^{+}=F_{m}^{-}$and the forcing coefficient $\beta_{0}=0$. For vertically forced Faraday waves, on the other hand, $g$ is constant in (5.9) and $\beta_{0} \neq 0$ with $\Omega=0$. This is a significant difference between horizontal and vertical oscillations. 


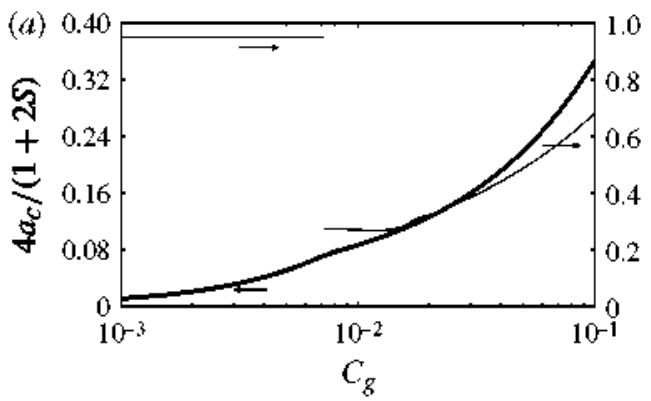

(b)
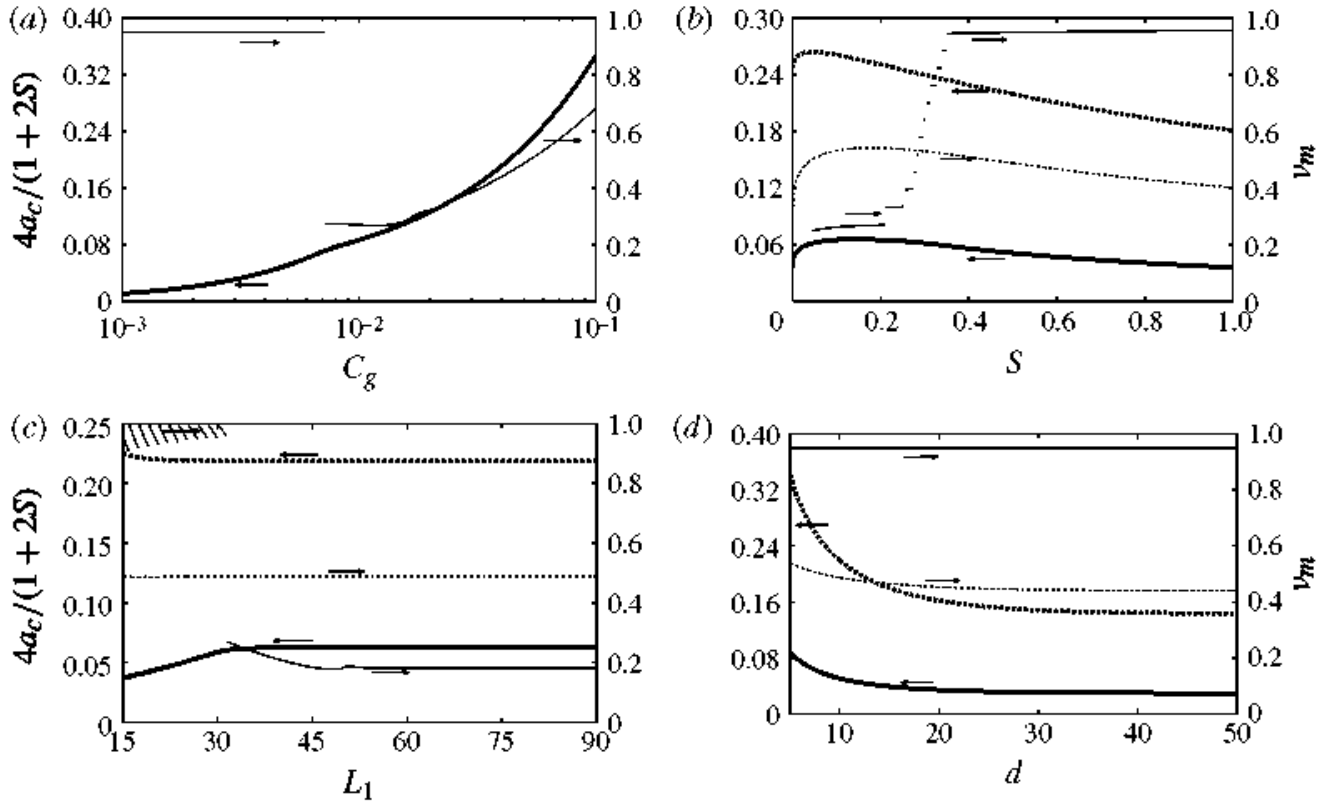

(d)

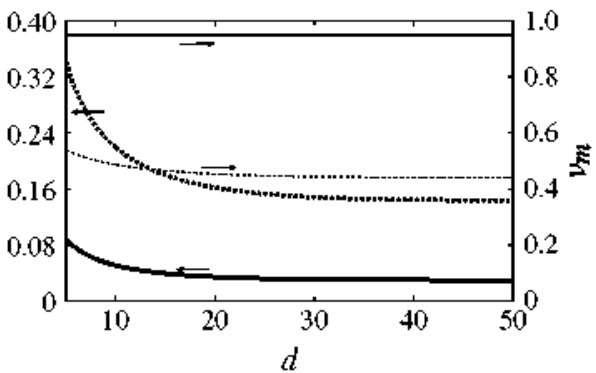

FIGURE 14. Instability threshold $a_{c}$ (thick lines) and the longitudinal wavenumber $v_{m}$ (thin lines) versus $(a) C_{8},(b) S,(c) L_{1}$ and $(d) d$, moving one parameter at a time for $C_{8}=0.005$ (solid lines) and 0.05 (dashed lines), $S=0.5, L_{1}=25$ and $d=10$. Arrows indicate the vertical scale for each curve.

\subsection{Dependence of the instability threshold $a_{c}$ on the non-dimensional parameters}

Since $L_{2}$ is large, the discrete threshold longitudinal wavenumber, $v_{m}$, is roughly that corresponding to the minimum value of $a$ along the marginal curve. Dependence of the instability threshold on the various parameters is now considered by applying the continuation process described in appendix $\mathrm{C}$ in a large region of the parameter space, defined as (cf. (5.1)) $5 \leqslant d \leqslant 50,15 \leqslant L_{1} \leqslant 90,0 \leqslant S \leqslant 1$ and $0.001 \leqslant C_{8} \leqslant 0.1$. In all cases, continuation is initiated at the point

$$
C_{g}=0.005 \text { and } 0.05, \quad S=0.5, \quad L_{1}=25, \quad d=10,
$$

and only one parameter is varied at a time. The instability threshold $a_{c}$ and the associated longitudinal wavenumber $v_{m}$ are provided in figure 14 , where we can see the following.

(a) As expected, $a_{c}$ increases as either $C_{g}$ is increased (which enhances viscous effects) or as $d$ is decreased (which decreases the amplitude of the OBF). The variation with $L_{1}$ and $d$ occurs mainly in the ranges $15 \leqslant L_{1} \leqslant 25$ and $5 \leqslant d \leqslant 40$, where $L_{1}$ and $d$ are somewhat comparable; for larger values of $L_{1}$ and $d$ the curves saturate. Dependence of $a_{c}$ on $S$ is not monotonic and peaks at some intermediate value of $S$, which depends on the remaining parameters.

(b) The orientation is generally oblique (namely, $0<v_{n t}^{c}<1$ ) except in two limits, both for quite small $C_{g}$. (i) For $S=0$, the orientation is perpendicular to the endwalls, which is consistent with the fact that standard gravitational crosswaves are perpendicular to the wavemakers, and confirms the conjecture in the Introduction that capillarity promotes obliqueness. (ii) For $S \geqslant 0.5$, the patterns 
are almost parallel to the vibrating endwalls, which was the limit implicit in the two-dimensional analysis by Varas \& Vega (2007).

(c) At some particular values of the parameters $C_{g}, S$ and $L_{1}, v_{m}$ shows abrupt transitions, which are due to phase slips in the $x$ direction; predicted and measured orientations may show large discrepancies in these regions.

The frequency $\Omega$ of the slow modulation, not plotted in figure 14 , is always very small, in the range $10^{-10}-10^{-2}$. Calculations clearly show that $\Omega \rightarrow 0$ as $L_{1} C_{g} \rightarrow \infty$ and $L_{1} / d \rightarrow \infty$, i.e. when the longitudinal length $L_{1}$ is large compared with both the viscous decay length of the waves and the horizontal extension of the OBF. In other words, $\Omega$ vanishes as interaction between the endwalls is weak. In the opposite limit, $\Omega$ increases as $L_{1}$ decreases, which suggests that strict $2: 1$ subharmonic oscillations $(\Omega=0)$ of individual sloshing modes $\left(L_{1} \sim 1\right)$ are not possible in rectangular containers under horizontal oscillation.

\section{Concluding remarks}

A general theory has been derived for the development of nearly inviscid, subharmonic waves in horizontally vibrated containers whose dimensions are large compared with the wavelength of the basic capillary-gravity waves. In this limit, a quasi-potential approximation derived in $\$ 2.1$ applies that greatly simplifies the formulation. The parametric instability that triggers the waves has been seen to be promoted by an oscillatory bulk flow that is uniquely determined by an inviscid linear problem formulated in $\$ 2.2$, whose solution is two-dimensional and can be used to calculate the parametric forcing term in closed form. Using these, a three-dimensional quasi-potential approximation was derived in $\S 3$ that provides the linear evolution of the subharmonic field. Taking advantage of the fact that this problem is singular, an appropriate solvability condition was applied that led a simpler two-dimensional equation for the free surface elevation of general subharmonic waves (consisting of arbitrary linear combinations of plane waves), with boundary conditions (derived in appendix B) that account for wave reflection at the sidewalls and endwalls. Using this simpler two-dimensional formulation and decomposing into normal modes in the transverse direction, an eigenvalue problem was obtained in $\S 4.1$. This problem shows that the instability is not strictly $2: 1$ subharmonic, but exhibits a second (small but non-zero) frequency that makes the resulting patterns generally quasi-periodic. The eigenvalue problem provides the instability threshold, the additional frequency, and the wave orientation at threshold. The resulting, generally oblique, quasi-periodic patterns were described, together with some secondary patterns resulting from mode interaction with nearby transverse modes (which is facilitated because the transverse size of the container is large) in $\$ 4.2$.

Comparison with measurements and visualizations in several experimental runs was made in $\$ 5.1$. Theoretical calculations showed very good quantitative agreement in connection with the instability threshold and reasonably good qualitative agreement in connection with the visualized patterns when such comparison was meaningful (reasonably close to threshold).

In addition, the theory developed in the paper was used in $\$ 5.2$ to elucidate the dependence of the instability threshold and the pattern orientation on the various non-dimensional parameters. In particular, it was seen that, except for gravity waves, the patterns are not perpendicular to the endwalls, but generally oblique and, moreover, align with the endwalls in some limits. This discussion can be used to make specific predictions. 
Let us now outline the unifying, general character of the theory developed above. This theory is the natural extension of the simplest case of vertical vibrations, in which the OBF equation is still (2.28) but the first boundary conditions in (2.29) and (2.30) must be replaced by $\partial_{x} P^{o b f}=0$ at $x= \pm L_{1}$ and $\partial_{z} P^{o b f}=4$ at $z=-d$ (note that the zero-pressure boundary condition in (2.30), which is specific for the OBF, is maintained). The resulting problem is solved with $P^{o b f}=4 z$ and the function $g$ appearing in (2.33) is set to $g(x)=1$. Substituting this into (4.11)-(4.12) gives a problem that can be solved in closed form to obtain the well-known Faraday instability threshold.

The theory can be naturally extended (in a relatively straightforward manner) for general vibrating systems with interfaces, provided that the counterparts of the assumptions (2.16) hold. This occurs if the forcing frequency is large compared with the first sloshing mode frequency and the vibrating acceleration and viscous effects are appropriately small. For instance, for oblique vibrations (neither horizontal, nor vertical), but still in the symmetry plane of the container parallel to the $x$ direction, the OBF remains independent of $y$ and the forcing function $g$ is readily calculated as

$$
g(x)=\sin \theta+\frac{4 \cos \theta}{\pi} \sum_{m>0, \text { odd }} \frac{\sinh [m \pi x /(2 d)]}{m \cosh \theta\left[m \pi L_{1} /(2 d)\right]},
$$

where $\theta$ is the angle of the vibrating direction and the $x$-axis. Substituting this into (4.11)-(4.13) and solving (numerically) the resulting problem provides the instability threshold. As a second example, for more general vibrating liquid systems with interfaces (such as vibrating drops), the $\mathrm{OBF}$ is still given by (2.28). The counterparts of the boundary conditions (2.29)-(2.30) consist in imposing that: (i) $P^{o b f}=0$ at the unperturbed free surface; and (ii) the wall-normal derivative of $P^{o b f}$ at the solid boundaries be equal to twice the normal velocities of the boundaries themselves. The resulting linear problem is always well-posed and provides the forcing pressure gradient at the free surface that must be used to obtain (using the counterpart of the quasi-potential approximation in \$2.1) the parametric forcing terms in the counterpart of (3.6)-(3.8). This latter problem applies in a $O(1)$-region near the unperturbed free surface and can be always reduced to a general, two-dimensional amplitude equation using curvilinear coordinates along the unperturbed free surface when this is non-flat, as with, e.g., vibrating drops.

We expect that the theory developed above will be a step further toward a correct description of the excitation of subharmonic waves in general vibrating systems with interfaces, identifying the parametric forcing mechanism and providing specific predictions for both the threshold acceleration and the resulting subharmonic patterns.

\section{Acknowledgements}

This research was partially supported by the Spanish Ministry of Science and Innovation, under Grants TRA2010-18054 (JMPG and JMV), MTM-2010-21135-C0202 (FV) and AYA2010-19081 (JP). We are indebted to two anonymous referees for their careful reading of the manuscript and for several comments on an earlier version of the paper that helped to improve the presentation of the results.

\section{Appendix A. Derivation of the evolution equations (2.24)}

For convenience, the evolution equation (2.24) are rewritten as

$$
\partial_{t} u^{u f s}=-\nabla p^{b l e}, \quad \partial_{t} w^{u f s}=-\partial_{z} p^{b l e}+2 C_{g} \Delta w^{u f s},
$$


where the superscripts ' $u f s$ ' and 'ble' denote hereafter the unperturbed free surface (where the velocity components will be calculated) and boundary-layer edge (just below the oscillatory boundary layer that is attached to the free surface) where the boundary conditions (2.23) apply. The first equation (A 1) readily follows from the horizontal momentum equation (2.5), anticipating that the pressure jump across the oscillatory boundary layer can be neglected in the approximation relevant here because

$$
p^{u f s}-p^{b l e}=O\left(\sqrt{C_{g}}\left|\boldsymbol{u}^{u f s} \cdot \nabla w^{u f s}\right|\right),
$$

which is obtained from (A 7) below, recalling that the thickness of the oscillatory boundary layer is $O\left(\sqrt{C_{g}}\right)$.

The second equation in (A 1), instead, does not coincide with the leading-order approximation of the vertical momentum equation (2.6) due to a jump of $\Delta w$ across the boundary layer, as is explained now. To the approximation relevant here, the continuity equation in (2.4) and the boundary condition (2.14) are written as

$$
\nabla \cdot \boldsymbol{u}+\partial_{z} w=0 \quad \text { if } z \leqslant 0, \quad \partial_{z} \boldsymbol{u}+\nabla w=\mathbf{0} \quad \text { at } z=0,
$$

which imply that $\partial_{z}(\nabla \cdot \boldsymbol{u})+\partial_{z z} w=0$ and $\boldsymbol{\nabla} \cdot\left(\partial_{z} \boldsymbol{u}\right)+\boldsymbol{\nabla} \cdot(\nabla w)=0$ at $z=0$. Eliminating $\partial_{z}(\boldsymbol{\nabla} \cdot \boldsymbol{u}) \equiv \boldsymbol{\nabla} \cdot\left(\partial_{z} \boldsymbol{u}\right)$ in these two equations yields

$$
\partial_{z z} w=\Delta w \quad \text { at } z=0 .
$$

This equation means that $\partial_{z z} w+\Delta w=2 \Delta w \neq 0$ at the free surface, while it vanishes in the bulk; thus, the above-mentioned jump. Substituting (A3)-(A4) into the vertical momentum equation in (2.6) leads to

$$
\partial_{t} w^{u f s}+2 \boldsymbol{u}^{u f s} \cdot \nabla w^{u f s}=-\partial_{z} p^{u f s}+2 C_{g} \Delta w^{u f s} \quad \text { at } z=0 .
$$

On the other hand, applying the operators $\nabla \cdot$ and $\partial_{z}$ to the momentum equations (2.5) and (2.6), respectively, and substituting (2.4) into the resulting equation lead to a standard Poisson equation for the pressure. Taking into account that $\left|\partial_{z}\right| \gg|\nabla|$ in the oscillatory boundary layer, the Poisson equation is written in first approximation as

$$
\partial_{z z} p=-\partial_{z}\left[\boldsymbol{u} \cdot\left(\nabla w-\partial_{z} \boldsymbol{u}\right)\right] .
$$

Integrating this equation across the oscillatory boundary layer and invoking (A 3) yield

$$
\partial_{z} p^{u f s}-\partial_{z} p^{b l e}=-2 \boldsymbol{u}^{u f s} \cdot \nabla w^{u f s},
$$

where it has been taken into account that $\nabla w-\partial_{z} \boldsymbol{u}=\mathbf{0}$ at the lower edge of the boundary layer (because vorticity vanishes there). Substituting (A 7) into (A 5) leads to the second equation (A 1), which completes the derivation.

\section{Appendix B. Wave reflection near the endwalls and sidewalls}

The analysis below is in the spirit of Hocking (1987) and Nicolas, Rivas \& Vega (1998); reflection of waves aligned with the endwalls (such as the harmonic waves, ignored here) was analysed in the context of horizontal vibrations by Varas \& Vega (2007).

Near the endwall $x=L_{1}$ (the endwall $x=-L_{1}$ and the sidewalls are treated similarly), the spatial variable $x$ is shifted and the $y$ Fourier transforms of the pressure and the free surface deflection are considered, as

$$
\hat{x}=x-L_{1}, \quad\left(P^{s}, F^{s}\right)=\int_{-1}^{1}(q, \phi) \mathrm{e}^{\mathrm{i} \kappa y} \mathrm{~d} \kappa .
$$


Substituting these into (3.9)-(3.11) (which are the relevant equations at leading order), the following equations and boundary conditions are obtained for the Fourier transforms $q$ and $\phi$

$$
\begin{gathered}
\partial_{x x} q+\partial_{z z} q-\kappa^{2} q=0 \quad \text { in } 0<\hat{x}<\infty, \quad-\infty<z<0, \\
\phi-\partial_{z} q=q-\left[(1-S)+S \kappa^{2}\right] \phi+S \partial_{x x} \phi=0 \quad \text { at } z=0, \\
\partial_{x} q=\phi=0 \quad \text { at } \hat{x}=0, \quad \partial_{z} q \rightarrow 0 \quad \text { as } z \rightarrow-\infty .
\end{gathered}
$$

If the boundary condition for $\phi$ at $\hat{x}=0$ is ignored, then a simple solution exists, namely

$$
\left(q_{1}, \phi_{1}\right)=\left(\mathrm{e}^{\tilde{z}}, 1\right) \cos v x
$$

where the longitudinal wavenumber $v$ is defined as

$$
v=\sqrt{1-\kappa^{2}} \text {. }
$$

In order to obtain a solution satisfying also the boundary condition for $\phi$, a second solution is calculated that does not satisfy this boundary condition either; the solution satisfying the boundary condition will be obtained as a linear combination of both.

Ignoring again the boundary condition for $\phi$ at $\hat{x}=0$, the equations and the remaining boundary conditions in (B 2)-(B 4) are solved via the Fourier cosine transform as

$$
q_{2}=\int_{0}^{\infty} B(\hat{\kappa}) \mathrm{e}^{\sqrt{\kappa^{2}+\hat{\kappa}^{2}}} \cdot \cos (\hat{\kappa} \hat{x}) \mathrm{d} \hat{\kappa}, \quad \phi_{2}=\int_{0}^{\infty} B(\hat{\kappa}) \sqrt{\kappa^{2}+\hat{\kappa}^{2}} \cos (\hat{\kappa} \hat{x}) \mathrm{d} \hat{\kappa},
$$

were $B(\hat{\kappa})$ is calculated as follows. First, this expression for $q_{2}$ is introduced into the second boundary condition (B 3). Integrating the resulting equation for $\phi$ yields

$$
\phi_{2}=C \mathrm{e}^{-\sqrt{\left(1-S v^{2}\right) / S} \dot{x}}+\int_{0}^{\infty} \frac{B(\hat{\kappa}) \cos (\hat{\kappa} \hat{x}) \mathrm{d} \hat{\kappa}}{1+S\left(\hat{\kappa}-v^{2}\right)} .
$$

where $C$ is an arbitrary constant and exponentially diverging behaviours of $\phi$ have been discarded. Identifying the two expressions for $\phi_{2}$ in (B 7) and (B 8) and taking into account that

$$
\mathrm{e}^{-\sqrt{\left(1-S v^{2}\right) / S} \dot{x}}=\frac{2}{\pi} \int_{0}^{\infty} \frac{\sqrt{\left(1-S v^{2}\right) / S} \cos (\hat{\kappa} \hat{x}) \mathrm{d} \hat{\kappa}}{\hat{\kappa}^{2}+\left(1-S v^{2}\right) / S}
$$

yield

$$
B(\hat{k})=\frac{2}{\pi} \frac{C \sqrt{S\left(1-S v^{2}\right)}}{\sqrt{1+\hat{\kappa}^{2}-v^{2}}\left[1+S\left(\hat{\kappa}-v^{2}\right)\right]-1},
$$

where all integrals must be understood hereafter in the sense of the principal value. Substituting this into (B 7) and (B 8) (this expression for $\phi_{2}$ exhibits better convergence properties than its counterpart in (B 7)) and setting $C=1$ lead to

$$
\begin{gathered}
q_{2}=\frac{2}{\pi} \int_{0}^{\infty} \frac{\sqrt{S\left(1-S v^{2}\right)} \mathrm{e}^{\sqrt{\kappa^{2}+\hat{\kappa}^{2}}} \cdot \cos (\hat{\kappa} \hat{x}) \mathrm{d} \hat{\kappa}}{\sqrt{1+\hat{\kappa}^{2}-v^{2}}\left[1+S\left(\hat{\kappa}^{2}-v^{2}\right)\right]-1}, \\
\phi_{2}=\mathrm{e}^{-\sqrt{\left(1-S v^{2}\right) / S} x}+\frac{2}{\pi} \int_{0}^{\infty} \frac{\sqrt{S\left(1-S v^{2}\right)} \cos (\hat{\kappa} \hat{x}) \mathrm{d} \hat{\kappa}}{\sqrt{1+\hat{\kappa}^{2}-v^{2}}\left[1+S\left(\hat{\kappa}-v^{2}\right)\right]^{2}-\left[1+S\left(\hat{\kappa}-v^{2}\right)\right]} .
\end{gathered}
$$


Now, once this second solution has been calculated, the general solution of (B 2)-(B 4), ignoring the boundary condition for $\phi$ at $\hat{x}=0$, is written as

$$
(q, \phi)=C_{1}\left(q_{1}, \phi_{1}\right)+C_{2}\left(q_{2}, \phi_{2}\right) .
$$

Invoking (B 5) and (B 12) and imposing the boundary condition that has been ignored so far (namely, $\phi=0$ at $\hat{x}=0$ ), it is seen that $C_{1}$ and $C_{2}$ must satisfy

$$
C_{1}=-C_{2} H_{1}\left(v^{2}, S\right),
$$

where the scalar $H_{1}$ is given by

$$
H_{1}=1+\frac{2}{\pi} \int_{0}^{\infty} \frac{\sqrt{S\left(1-S v^{2}\right)} \mathrm{d} \hat{\kappa}}{\sqrt{1+\hat{\kappa}^{2}-v^{2}}\left[1+S\left(\hat{\kappa}^{2}-v^{2}\right)\right]^{2}-\left[1+S\left(\hat{\kappa}^{2}-v^{2}\right)\right]} .
$$

Substituting (B 14) into (B 13) and setting, e.g., $C_{2}=-1$, a solution of (B 2)-(B 4) is obtained that shows the asymptotic behaviour (up to exponentially small terms)

$$
\phi=H_{1}\left(v^{2}, S\right) \cos v \hat{x}+\frac{2 \sqrt{S\left(1-S v^{2}\right)} \sin v \hat{x}}{(1+2 S) v} \text { as } \hat{x} \rightarrow \infty .
$$

This behaviour follows by noting in (B 12) that $\int_{0}^{\infty}\left(v^{2}-\hat{\kappa}^{2}\right)^{-1} \cos (\hat{\kappa} \hat{x}) \mathrm{d} \hat{\kappa}=$ $\pi \sin (v \hat{x}) /(2 v)$ and that if $\hat{\kappa}^{2} g(\hat{\kappa})$ is bounded, then $\int_{0}^{\infty} g(\hat{\kappa}) \cos (\hat{\kappa} x) \mathrm{d} \hat{\kappa} \rightarrow 0$ exponentially as $\hat{x} \rightarrow \infty$. The limit (B 16) can also be written as

$$
\phi=\sin \left[v \hat{x}-\alpha\left(v^{2}, S\right)\right] \quad \text { as } \hat{x} \rightarrow \infty,
$$

where the phase shift $\alpha$ is given by

$$
\alpha\left(v^{2}, S\right)=\tan ^{-1} \frac{(1+2 S) v H_{1}\left(S, v^{2}\right)}{2 \sqrt{S\left(1-S v^{2}\right)}} .
$$

Still, the behaviour (B 17) can also be written as

$$
\partial_{x} \phi-\gamma \phi=0 \quad \text { as } \hat{x} \rightarrow \infty,
$$

where (also using (B 6))

$$
\gamma\left(\kappa^{2}, S\right)=\frac{v}{\tan \alpha}=\frac{2 \sqrt{S\left(1-S+S \kappa^{2}\right)}}{(1+2 S) H_{1}\left(1-\kappa^{2}, S\right)} .
$$

For illustration, the scalar $\gamma$ is plotted in figure 15 versus $|\kappa|$ for various representative values of $S$, as indicated.

Matching with the outer solution, outside the layer analysed in this appendix, and invoking (B 1) provides the following boundary condition for the solution in the bulk of the container

$$
\partial_{x} F-\int_{-1}^{1} \gamma(S, \kappa) \phi(\kappa) \mathrm{e}^{\mathrm{i} \kappa y} \mathrm{~d} \kappa=0 \quad \text { at } x=-L_{1},
$$

where $\phi$ is the Fourier transform of $F\left(L_{1}, y\right)$, defined such that

$$
F\left(L_{1}, y\right)=\int_{-1}^{1} \phi(\kappa) \mathrm{e}^{\mathrm{i} \kappa y} \mathrm{~d} \kappa .
$$

The strictly inviscid, unforced approximation above ignores both a Stokes boundary layer attached to the endwall and higher-order terms resulting from products of $a \ll 1$ 


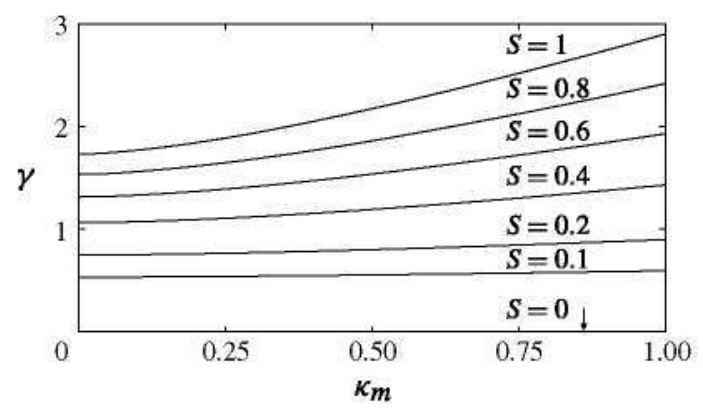

FIGURE 15. The scalar $\gamma$ appearing in (B 20).

and the flow variables. If all of these were taken into account, a fairly involved analysis (omitted here) would include higher-order terms in the boundary condition (B 21), which would read

$$
\partial_{\hat{x}} F-\int_{-1}^{1}\left[\left(\gamma+\sqrt{\mathrm{i} C_{g}} \gamma_{1}\right) \phi+a \gamma_{1} \bar{\phi}\right] \mathrm{d} \kappa=0 \quad \text { at } \hat{x}=0,
$$

for some real coefficients $\gamma_{1}$ and $\gamma_{2}$ that are $O(1)$. Nevertheless, the $O\left(\sqrt{C_{g}}\right)$ coefficient $\gamma_{1}$ is expected to be somewhat small due to the fact that the contact line is pinned; this has been theoretically shown by Higuera, Nicolas \& Vega (1994) and Martel, Nicolás \& Vega (1998) for the first sloshing modes in various fluid configurations and experimentally checked by Howell et al. (2000). Of course, this cannot be true in the strict gravity wave limit, $S=0$, but the additional term can still be neglected under the last assumption imposed in (2.16). Similarly, since the OBF extends a distance $d \gg 1$ in the bulk, the parametric forcing term in (B 23) can be neglected compared with the parametric forcing effect of the OBF. It is interesting to note that this term provides the parametric forcing effect of the vibrating endwalls acting as wave makers, which would be the only source for parametric forcing if the $\mathrm{OBF}$ were not present.

\section{Appendix C. Numerical methods}

Reflection symmetric solutions of (4.11)-(4.13) satisfying (4.16) are calculated using two different methods.

(i) A spectral method resulting from a truncated expansion of $F_{m}^{ \pm}$into harmonic functions satisfying the boundary conditions (4.13). This yields a homogeneous, linear system of algebraic equations for the complex mode amplitudes. Requiring that this system exhibits non-trivial solutions, a complex solvability condition is obtained that provides the real unknowns $\Omega$ and $a$ in terms of $v_{m}$ (namely, the functions (4.17)).

(ii) A multiple shooting method considering solutions in the half subdomain $0 \leqslant x \leqslant$ $L_{1}$ that satisfy the boundary condition (4.13) and (cf. (4.16))

$$
\partial_{x} F_{m}^{+}=F_{m}^{-}=0 \quad \text { at } x=0 .
$$

The resulting initial value problem is integrated from $x=L_{1}$ to $x=0$ (to avoid ill conditioning), with two linearly independent initial conditions at $x=L_{1}$ satisfying the boundary conditions (4.13); the weak (logarithmic) singularity of the 
function $g$ mentioned after (2.34) is handled by appropriate $x$-stepping. Imposing (C 1 ) on a linear combination of the two solutions leads to a $2 \times 2$ homogenous linear system on the complex coefficients of the linear combination. Requiring that this system exhibits non-trivial solutions leads to complex equation that determines the real unknowns $\Omega$ and $a$.

In both cases, a numerical pseudo-arclength continuation method (Allgower \& Georg 2003 ) is applied to follow the neutral stability curves considered in $\$ 5$.

\section{REFERENCES}

ALlgower, E. L. \& GEoRg, K. 2003 Introduction to Numerical Continuation Methods. SIAM Classics in Applied Mathematics, vol. 45. Society for Industrial and Applied Mathematics.

Barnard, B. J. S. \& Pritchard, W. G. 1972 Cross-waves. Part 2. Experiments. J. Fluid Mech. 55. $245-255$.

BENIAMIN, T. B. \& URSELL. F. 1954 The stability of the plane free surface of a liquid in vertical periodic motion. Proc. R. Soc. Lond. A 225, 505-515.

BERNOFF, A. J., KWOK, L. P. \& LICHTER, S. 1989 Viscous cross-waves: an analytical treatment. Phws. Fluids A 1, 678-688.

Faltinsen, O. M., Rognebakke, O. F. \& Timokha, A. N. 2006 Transient and steady-state amplitudes of resonant three-dimensional sloshing in a square-based tank with a finite fluid depth. Phys. Fluids 18, 012103.

FARADAY, M. 1831 On the forms and states assumed by fluids in contact with vibrating elastic surfaces. Phil. Trans. R. Soc. Lond. 121, 319-340.

Fauve, S. 1995 Parametric instabilities. In Dynamics of Nonlinear and Disordered Systems (ed. G. Martínez-Mekler \& T. H. Seligman), pp. 67-115. World Scientific.

FENG, Z. C. 1997 Transition to travelling waves from standing waves in a rectangular container subjected to horizontal excitations. Phys. Rev. Lett. 79, 415-418.

Frandsen. J. B. 1997 Sloshing motions in excited tanks. J. Comput. Phys. 196, 53-87.

Funa Koshi, M. \& INOUE, S. 1988 Surface waves due to resonant horizontal oscillation. $J$. Fluid Mech. 192, 219-247.

Garrett, C. J. R. 1970 On cross-waves. J. Flitd Mech. 41, 837-849.

GONZÁlEZ-ViÑAs, W. \& SALAM, J, 1994 Surface waves periodically excited in a $\mathrm{CO}_{2}$ tube. Europhys. Lett. 26. 665-670.

GuCKenHeIMER, J. \& HoLmes. P. 1983 Nontinear Oscillations Dynanical Systems and Bifurcation of Vector Fields. Springer.

Havelock, T. H. 1929 Forced surface waves on water. Phil. Mag. 8, 569-576.

HermanN, M. \& Timokha, A. 2008 Modal modelling of the nonlinear resonant fluid sloshigh in a rectangular tank II: secondary resonance. Math. Models Meth. Appl. Sci. 18, 1845-1867.

Higuera, M., Nicolas, J. A. \& VEGa, J. M. 1994 Linear oscillations of weakly dissipative axisymmetric liquid bridges. Phys. Fluids 6, 438-450.

HILL. D. F. 2003 Transient and steady state amplitudes of forced waves in rectangular basins. Phys. Fluids 15, 1576-1587.

Hocking. L. M. 1987 Reflection of capillary-gravity waves. Wave Motion 9, 217-226.

Howell, D., Heath, T., McKenna, C., Hwang, W. \& Schatz, M. F. 2000 Measurements of surface-wave damping in a container. Phys. Fluids 12, 322-326.

JONES, A. F. 1984 The generation of cross-waves in a long deep channel by parametric resonance. J. Fluid Mech. 138. 53-74.

Joseph, D., Funada, T. \& WANG, J. 2007 Potential Flows of Viscous and Viscoelastic Fluids. Cambridge University Press.

KuZnetsov, Y, A, 1998 Elentents of Applied Bifurcation Theory. Springer.

Martel, C., Nicolás, J. A. \& VegA, J. M. 1998 Surface-wave damping in a brimful circular cylinder. J. Fluid Mech. 360, 213-228. See also Corrigendum. J. Fluid Mech. 373, 379. 
MCGoLdRICK, L. F. 1965 Resonant interactions among capillary-gravity waves. J. Fluid Mech. 21, 305-331.

MILES, J. W. 1984 Resonantly forced surface waves in a circular cylinder. J. Fluid Mech. 149, $15-31$.

Miles, J. \& Henderson, D. 1990 Parametrically forced surface waves. Annu. Rev. Fluid Mech. 22. $143-165$.

MoISY. F., RABAUD, M. \& SLASAC. K. 2009 A synthetic Schlieren method for the measurement of the topography of a liquid interface. Exp. Fluids 46. 1021-1036.

Moisy, F., Michon, G. J., Rabaud, M. \& Sultan, E. 2012 Cross-waves induced by the vertical oscillation of a fully immersed vertical plate. Phws. Fluids 24, 022110.

Nicolas, J. A., Rivas, D. \& Vega, J. M. 1998 On the steady streaming flow due to high-frequency vibration in nearly inviscid liquid bridges. J. Flaid Mech 147-156.

Porter, J., Tinao, I., Laveron-Simavilla, A. \& Lopez, C. A. 2012 Pattem selection in a horizontally vibrated container. Fluid Dyn. Res. 44, 065501.

Shemer, L. \& Lichter, S. 1990 The mode number dependence of neutral stability cross-waves. Exp. Fluids 9, 148-152.

Shemer, L. \& Chamesse, M. 1999 Experiments on nonlinear gravity-capillary waves. J. Fluid Mech. 380. 205-232.

TANEDA, S. 1994 Visual observations of the flow around a half-submerged oscillating circular cylinder. Fluid Dyn. Res. 13, 119-151.

VARAS, F. \& VEGA, J. M. 2007 Modulated surface waves in large aspect ratio, horizontally vibrated containers. J. Fluid Mech. 579. 271-304.

VEGA, J. M. RÜDIGER, S. \& VIÑAlS. J. 2004 Phenomenological model of weakly damped Faraday waves and the associated mean flow. Phys. Rev. E 70.0406306.

VUKASINovic, B.. SMITH. B. \& GLEZER, A. 2007 Dynamics of a sessile drop in forced vibration. J. Fluid Mech. 587, 395-423.

Wu, C. H. \& CHEN, B. F. 2009 Sloshing waves and resonance modes of fluid in a 3D tank by a time-independent finite difference method. Ocean Engng 36, 500-510.

ZHANG, W. \& ViÑALS, J. 1997 Pattern formation in weakly damped parametric surface waves. J. Fluid Mech. 336. 301-330. 